\title{
Probing the Donor and Acceptor Substrate Specificity of the $\gamma$-Glutamyl Transpeptidase
}

\author{
Xin $\mathrm{Hu},{ }^{*}, \star \|$ Patricia M. Legler, ${ }^{*}$, Ilja Khavrutskii, ${ }^{\dagger}$ Angelo Scorpio, ${ }^{\S}$ Jaimee R. Compton, ${ }^{\ddagger}$ \\ Kelly L. Robertson, ${ }^{\ddagger}$ Arthur M. Friedlander, ${ }^{\S}$ and Anders Wallquist ${ }^{\dagger}$
}

\author{
${ }^{\dagger}$ Biotechnology HPC Software Applications Institute, Telemedicine and Advanced Technology Research Center, U.S. Army Medical \\ Research and Materiel Command, Fort Detrick, Maryland 21702, United States \\ ${ }^{\ddagger}$ Naval Research Laboratories, Washington, D.C. 20375, United States \\ ${ }^{\S}$ U.S. Army Medical Research Institute of Infectious Diseases, Fort Detrick, Maryland 21702, United States
}

Supporting Information

\begin{abstract}
Glutamyl transpeptidase (GGT) is a twosubstrate enzyme that plays a central role in glutathione metabolism and is a potential target for drug design. GGT catalyzes the cleavage of $\gamma$-glutamyl donor substrates and the transfer of the $\gamma$-glutamyl moiety to an amine of an acceptor substrate or water. Although structures of bacterial GGT have revealed details of the protein-ligand interactions at the donor site, the acceptor substrate site is relatively undefined. The recent identification of a species-specific acceptor site inhibitor, OU749, suggests that these inhibitors may be less toxic than glutamine analogues. Here we investigated the donor and acceptor substrate preferences of Bacillus anthracis GGT

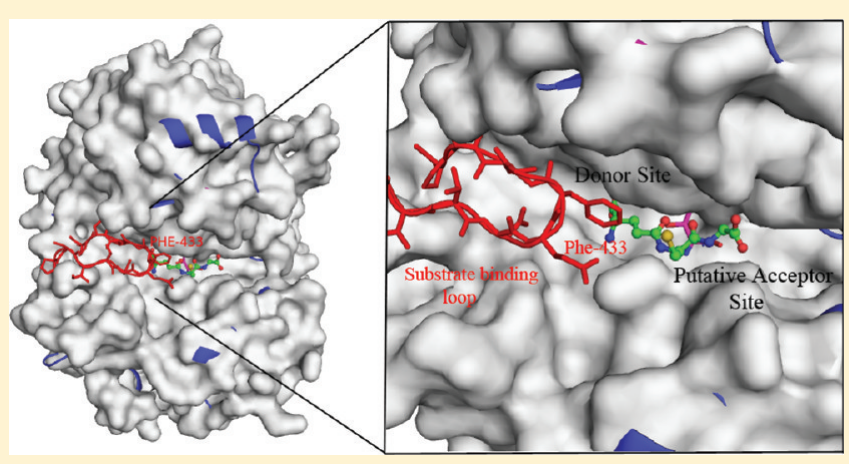
(CapD) and applied computational approaches in combination with kinetics to probe the structural basis of the enzyme's substrate and inhibitor binding specificities and compare them with human GGT. Site-directed mutagenesis studies showed that the R432A and R520S variants exhibited 6- and 95-fold decreases in hydrolase activity, respectively, and that their activity was not stimulated by the addition of the L-Cys acceptor substrate, suggesting an additional role in acceptor binding and/or catalysis of transpeptidation. Rat GGT (and presumably HuGGT) has strict stereospecificity for L-amino acid acceptor substrates, while CapD can utilize both L- and D-acceptor substrates comparably. Modeling and kinetic analysis suggest that R520 and R432 allow two alternate acceptor substrate binding modes for L- and D-acceptors. R432 is conserved in Francisella tularensis, Yersinia pestis, Burkholderia mallei, Helicobacter pylori and Escherichia coli, but not in human GGT. Docking and MD simulations point toward key residues that contribute to inhibitor and acceptor substrate binding, providing a guide to designing novel and specific GGT inhibitors.
\end{abstract}

$\gamma$-Glutamyl transpeptidase (GGT, EC 2.3.2.2) is an enzyme that plays a central role in glutathione metabolism and cysteine salvage. ${ }^{1-3}$ It has been found in a wide variety of organisms, including mammals, plants, and bacteria. The latter includes such pathogens as Helicobacter pylori, Escherichia coli, Burkholderia mallei, Staphylococcus aureus, Yersinia pestis, Bacillus anthracis, and Francisella tularensis. ${ }^{1,2}$ In humans, GGT (HuGGT) is implicated in a number of physiological processes and disorders, such as tumor progression, drug resistance, and neurodegenerative diseases. ${ }^{4-6}$ The bacterial GGT enzyme in H. pylori (HpGGT) is an apoptosis-inducing protein and is essential for the establishment of infection. ${ }^{7,8}$ The B. anthracis GGT (CapD) is a virulence factor required in anchoring the capsule to the bacterial cell wall as well as capsule remodeling. ${ }^{9}$ CapD is an unusual GGT in that it has been shown to be unable to hydrolyze glutathione $(\mathrm{GSH}) ;^{3}$ its endogenous substrate is likely the poly- $\gamma$-D-glutamic acid (PDGA) capsule surrounding the bacterium. The capsule allows $B$. anthracis to evade the immune system by preventing phagocytic killing by immune cells and by acting as a physical barrier to surface antigens. The capsule itself is weakly immunogenic. ${ }^{10}$ CapD is not a typical antimicrobial target in that it is not a bactericidal target. However, inhibition of CapD results in the loss of the capsule from the cell wall, resulting in unencapsulated bacteria that are now susceptible to phagocytosis by neutrophils and can be cleared by the immune system. Moreover, CapD is a membrane-associated enzyme, ${ }^{3}$ and inhibitors of the enzyme could be used to bypass some mechanisms of antimicrobial resistance (e.g., efflux pumps). GGTs of Gram-negative bacteria are also potential antimicrobial targets as host glutathione can function as a source of cysteine. Mutant strains of F. tularensis that lack functional GGT have been shown to have impaired intracellular replication and are strongly attenuated for

Received: June 27, 2011

Revised: January 17, 2012

Published: January 17, 2012 
virulence in mice. ${ }^{11}$ Therefore, GGT has been identified as a valid target for both anticancer and antimicrobial therapeutics.

GGTs catalyze the cleavage of $\gamma$-glutamyl donor substrates (e.g., glutathione and its S-conjugates), and the transfer of the $\gamma$-glutamyl group to an acceptor substrate amino acid, $\mathrm{N}$-terminal residue of a polypeptide (transpeptidation), or water (hydrolysis) following a ping-pong kinetic mechanism. ${ }^{12,13}$ GGT is expressed as an $\sim 60 \mathrm{kDa}$ precursor and is activated by an autocatalytic internal cleavage (e.g., $\mathrm{S} \downarrow \underline{T T H F V}$ in CapD and $\mathrm{G} \downarrow$ TAHLS in HuGGT) to generate a heterodimer consisting of $\sim 40$ and $\sim 20 \mathrm{kDa}$ subunits. The $\mathrm{N}$-terminal threonine (underlined) hydroxyl of the small subunit acts as the nucleophile in both the autoprocessing and enzyme-catalyzed reactions. ${ }^{1,2}$

A close homologue of HuGGT, namely, rat GGT that is 79\% identical to human GGT, can utilize a number of acceptor substrates in a $\mathrm{pH}$-dependent fashion. Typical transpeptidase acceptor substrates have a terminal amino group. The simplest acceptor substrates include single amino acids and glycine dipeptides (Gly-Ala or Z-Gly, where Z is W, F, Y, H, A, Q, C, $\mathrm{M}, \mathrm{S}, \mathrm{L}, \mathrm{K}, \mathrm{A}$, or $\mathrm{G}){ }^{13}$ The product of the GGT-catalyzed hydrolysis of glutathione, Cys-Gly, mimics both the donor substrate and dipeptide acceptor substrates. The acceptor substrate can therefore exploit features of the binding site that stabilize binding of glutathione on the acceptor side. Cook et al. recognized that the $\mathrm{p} K_{\mathrm{a}}$ of the $\alpha$-amino group of glycyl dipeptides is significantly lower than those of free amino acids and may in part explain why glycyl dipeptides tend to be better acceptor substrates for rat GGT than free amino acids. The transpeptidation reaction has a bell-shaped $\mathrm{pH}$-rate profile for both rat GGT and HuGGT, ${ }^{14,16-18}$ but only an ascending limb in E. coli GGT. ${ }^{19}$ The hydrolysis reaction yields a relatively flat $\mathrm{pH}$-rate profile. While free amino acids are typically found in their zwitterionic forms, the reactive form of the acceptor substrate is believed to contain a neutral terminal amine as the $\mathrm{p} K_{\mathrm{a}}$ of the ascending limb varies with the acceptor. ${ }^{15}$ On the acceptor substrate side, a positively charged residue with a $\mathrm{p} K_{\mathrm{a}}$ of 8.46 in rat GGT (a similar $\mathrm{pH}$-rate profile was reported for $\mathrm{HuGGT}^{18}$ ) was proposed to be important for binding the acceptor and/or catalysis; ${ }^{15}$ however, a specific residue has not been shown to account for the descending limb of the $\mathrm{pH}$-rate profile. Herein, we identify an important mobile electrostatic catalyst, Arg-520 in CapD (Lys-562 in HuGGT), which may interact with the acceptor substrate and contribute to catalysis.

X-ray structures of GGTs from several bacterial species have revealed atomic details of protein-donor substrate interactions ${ }^{20-25}$ and the acyl intermediate. ${ }^{20}$ Despite a high degree of conservation of structure and function between human and bacterial GGT homologues, significant differences in acceptor substrate and inhibitor preferences are evident. For example, rat and E. coli GGT have strict stereospecificity for L-amino acid acceptor substrates, ${ }^{26,27}$ while $B$. anthracis CapD does not (this paper). HuGGT also catalyzes transpeptidation 180-fold faster than hydrolysis, ${ }^{28,29}$ while HpGGT shows a modest 2 -fold ${ }^{30}$ and CapD a 12-fold difference (this paper) between the rates. Residues, which account for the faster rate transpeptidation versus hydrolysis, and acceptor substrate stereospecificity have not been previously identified. We hypothesized that differences may arise from nonconserved residues in the relatively undefined acceptor substrate binding site.

Within the putative acceptor substrate site, two alternate side chain conformers were observed for Arg-520 in the X-ray crystal structure of CapD [Protein Data Bank (PDB) entry $\left.3 \mathrm{G} \mathrm{K}^{25}\right]$. To determine if the flexibility of this and other residues may also account for differences in substrate binding and/or product release, we examined molecular dynamics simulations of bacterial and human GGTs.

Human GGT is a complementary drug target for various glutathione-sensitive cancer therapies. ${ }^{31-34}$ Although a number of potent inhibitors of HuGGT have been discovered in the past, most modify the enzyme irreversibly and have associated toxicities. Richter et al. also identified an inhibitor for CapD, Capsidin, ${ }^{9}$ which irreversibly acylates the active site threonine. While effective in inhibiting the enzyme in vitro, the acetyl groups of Capsidin were readily hydrolyzed by serum carboxylesterases. The human GGT inhibitors, which bind in the donor substrate site and mimic glutamate, such as acivicin, inhibit CapD poorly ( $\mathrm{IC}_{50}>20 \mathrm{mM}$ ) (data not shown) and cannot be repurposed as antimicrobials. Recently, a novel class of HuGGT inhibitor OU749 was identified. ${ }^{34}$ Unlike donor substrate analogue inhibitors, OU749 is believed to bind the acceptor site and is an uncompetitive inhibitor with respect to the $\gamma$-glutamyl donor substrate. Consequently, OU749 is significantly less toxic than acivicin and exhibits strong species-specific GGT inhibition. ${ }^{34}$ The discovery of OU749 suggested a promising direction toward the design of nontoxic, species-selective, acceptor site inhibitors of GGT, which may extend to bacterial GGT.

In this study, we applied computational and kinetic approaches to investigate both the donor and acceptor substrate specificities of $B$. anthracis CapD and compare them to those of HuGGT to identify any unique features of the bacterial enzyme's active site that could be exploited in followon in silico drug screening. In addition to natural acceptor and donor substrates and their derivatives, we examined the uncompetitive acceptor site inhibitor OU749 as a molecular probe to further explore the structural basis of inhibitor binding. One surprising commonality between rat GGT (and presumably HuGGT, which is $79 \%$ identical) and CapD is that both GGTs share the same acceptor substrate (L-Cys) preference (this paper) but differ in their binding specificity for OU749. To begin to understand species-specific inhibition within the acceptor substrate site, we determined the optimal binding conformation of selected substrates and inhibitors using a multistage docking and refinement strategy. Molecular dynamic simulations, site-directed mutagenesis, and kinetic analysis were used to characterize key residues at the putative acceptor substrate site that may account for species-specific acceptor substrate and acceptor site inhibitor binding specificities.

\section{EXPERIMENTAL PROCEDURES}

B. anthracis CapD Cloning, Protein Expression, and Purification. The CapD expression construct expressed a maltose-binding protein (MBP) fusion protein with a Factor Xa cleavage site prior to the CapD sequence. The first 27 residues were predicted to be the signal peptide sequence and were excluded. $^{35}$ The insert was placed between the XmnI and HindIII cleavage sites of the pMAL-c2 plasmid (New England Biolabs, Ipswich, MA) using the forward primer, ${ }^{5} \mathrm{CAGC}$ GAAGGATTTCTTTCAATAAAATAAAAGACAGTGTTA ${ }^{3}$, and the reverse primer, ${ }^{5}$ CAGCAAGCTTCTATTTATTTGATTTCCAAGTTCCATT ${ }^{3 \prime}$. The protein was expressed in E. coli BL-21(DE3) cells at $17^{\circ} \mathrm{C}$ for $20 \mathrm{~h}$. Cells were lysed in $1 \times$ phosphate-buffered saline (PBS) ( $\mathrm{pH} 7.4), 5 \%$ glycerol, $0.3 \mathrm{mg} / \mathrm{mL}$ lysozyme, and $0.05 \mathrm{mg} / \mathrm{mL}$ DNAase and sonicated. 
The lysate was centrifuged for $30 \mathrm{~min}$ at $20000 \mathrm{~g}$, and the supernatant was loaded onto an amylose-resin column equilibrated with $1 \times$ PBS ( $\mathrm{pH} 7.4)$. The column was eluted with $1 \times$ PBS $(\mathrm{pH} 7.4)$ and $10 \mathrm{mM}$ maltose. The protein was dialyzed into $0.5 \times$ PBS $(\mathrm{pH} 7.4)$ and loaded onto a $\mathrm{Q}-$ Sepharose column. The protein was eluted with a gradient of sodium chloride $(0$ to $1.0 \mathrm{M})$. Fractions were combined and dialyzed against $0.5 \times$ PBS ( $\mathrm{pH} 7.4)$ and loaded onto an SPSepharose column equilibrated with the same buffer. The protein was eluted with a gradient of sodium chloride (0 to $1.0 \mathrm{M}$ ). The protein was concentrated, and the buffer was exchanged into $1 \times$ PBS ( $\mathrm{pH} 7.4$ ) and $50 \%$ glycerol and stored at $-20{ }^{\circ} \mathrm{C}$. For assays, the glycerol was removed using a PD-10 column equilibrated with assay buffer.

Expression of B. anthracis CapD Protein and Purification of Site-Directed Mutants. Three site-directed mutants, R520S, R432A, and N373A, were constructed by Geneart Inc. (Regensburg, Germany) using the pMAL plasmid. All were purified using the same procedure as for wild-type (WT) CapD-MBP described above.

B. anthracis CapD Assays. Capsule degradation was observed by gel electrophoresis using a 0.5 to $1.0 \%$ agarose gel in $1 \times$ TAE buffer (40 mM Tris-acetate and $1 \mathrm{mM}$ EDTA). The capsule was purified from the Ames strain of B. anthracis as described previously. ${ }^{36}$ The gel was stained with Loeffler's methylene blue solution $(0.3 \mathrm{~g}$ of methylene blue, $30 \mathrm{~mL}$ of ethanol, and $100 \mathrm{~mL}$ of water). Reaction mixtures contained $100 \mathrm{mM}$ Tris ( $\mathrm{pH} 8.0)$, capsule, CapD-MBP $(25 \mu \mathrm{g} / \mathrm{mL})$, and acceptor $(0.5 \mu \mathrm{g} / \mathrm{mL})$ and were incubated for $30 \mathrm{~min}$ at $37^{\circ} \mathrm{C}$. The capsular material was used at a final concentration of $0.8-$ $1.0 \mu \mathrm{g} / \mu \mathrm{L}$ for assays and was held at a constant concentration in an experiment.

Steady state kinetic parameters were measured using a synthesized FRET donor substrate, Abz- $\gamma \gamma$-D-Glu $]_{5}$-Dnp, described by Richter et al. ${ }^{9}$ The FRET donor substrate is a 5-mer of poly-D-glutamate with an $\mathrm{N}$-terminal 2-aminobenzoyl $(\mathrm{Abz})$ fluorophore and C-terminal 2,4-dinitrophenyl (DNP) quencher. The donor substrate was synthesized by BioPeptide Co. Inc. (San Diego, CA). Initial velocities were measured by varying the concentration of the FRET donor substrate and acceptor substrate. Similar kinetic analyses of GGT have been conducted by others. ${ }^{26,37}$ Initial velocities were globally fit to the velocity equation for a ping-pong bi-bi kinetic mechanism:

$$
v=\frac{V_{\max }[\mathrm{S}][\mathrm{A}]}{K_{\mathrm{ma}}[\mathrm{S}]+K_{\mathrm{ms}}[\mathrm{A}]+[\mathrm{S}][\mathrm{A}]}
$$

where $[S]$ is the concentration of the FRET donor substrate, $[\mathrm{A}]$ is the concentration of the acceptor substrate, $K_{\mathrm{ma}}$ is the Michaelis constant for the acceptor substrate, and $K_{\mathrm{ms}}$ is the Michaelis constant for the donor substrate. The $K_{\mathrm{ms}}$ of the donor substrate is an apparent $K_{\mathrm{m}}$ as the acceptor can competitively inhibit the enzyme. ${ }^{13,26}$ Rates were measured in $25 \mathrm{mM}$ HEPES ( $\mathrm{pH} 7.4$ ) and $0.1 \%$ Tween 20 at $22 \pm 3{ }^{\circ} \mathrm{C}$ using an excitation wavelength of $315 \mathrm{~nm}$ and an emission wavelength of $415 \mathrm{~nm}$. Data were corrected for inner filter effects using the same substrate lacking the quencher. Data were fit using Grafit version 6.0.1 (Erithacus Software Ltd.). All chemicals were purchased from Sigma (St. Louis, MO).

Expression and Purification of HuGGT. The HuGGT expression vector was a kind gift from M. Hanigan (University of Oklahoma, Oklahoma City, OK). COS-1 cells were plated in three $75 \mathrm{~cm}^{2}$ tissue culture flasks in $10 \mathrm{~mL}$ of Dulbecco's modified minimum essential medium (DMEM) (Cellgro) containing $10 \%$ fetal bovine serum (Cellgro) at $37{ }^{\circ} \mathrm{C}$ in the presence of $5 \% \mathrm{CO}_{2}$. The following day, the flasks, at approximately $80 \%$ confluence, were transfected with $5.5 \mu \mathrm{g}$ of the pcDNA3.1(+) HuGGT plasmid and $20 \mu \mathrm{L}$ of Lipofectamine 2000 (lipofectamine) (Life Technologies), according to the manufacturer's recommendations. Following a $48 \mathrm{~h}$ incubation, the cells were washed twice with ice-cold $1 \times$ PBS and then harvested by being scraped. The cells were centrifuged at $1000 \mathrm{rpm}$ and $4{ }^{\circ} \mathrm{C}$ for $15 \mathrm{~min}$, and the pellet was flash-frozen in liquid nitrogen. Cells were lysed in $1.5 \mathrm{~mL}$ of lysis buffer [ $50 \mathrm{mM}$ HEPES ( $\mathrm{pH} 7.4$ ), $150 \mathrm{mM} \mathrm{NaCl}, 0.5 \%$ CHAPS, and 1 EDTA-free protease tablet (Roche) per $2.5 \mathrm{~mL}$ of lysis buffer] for $30 \mathrm{~min}$ with rocking. Protein was dialyzed into $500 \mathrm{~mL}$ of $50 \mathrm{mM}$ HEPES ( $\mathrm{pH} 8.0$ ), $150 \mathrm{mM} \mathrm{NaCl}$, with $1.0 \mathrm{~mL}$ of uncharged Chelating Sepharose (GE Healthcare) to remove any metal ions and the detergent. Protein was loaded onto a Chelating Sepharose column (GE Healthcare) charged with nickel and equilibrated with $50 \mathrm{mM}$ HEPES ( $\mathrm{pH}$ 8.0) and $500 \mathrm{mM} \mathrm{NaCl}$, and the column was washed with 2 column volumes of buffer after loading. The protein was eluted with the same buffer containing $300 \mathrm{mM}$ imidazole. Buffer was exchanged (five times) to $50 \mathrm{mM}$ HEPES ( $\mathrm{pH} 8.0$ ) and 150 $\mathrm{mM} \mathrm{NaCl}$ using an ultrafiltration unit. Protein was flash-frozen in liquid nitrogen and stored at $-80{ }^{\circ} \mathrm{C}$ until it was assayed.

HuGGT Assays. Steady state kinetic parameters were measured in $50 \mathrm{mM}$ HEPES $(\mathrm{pH} 8.0)$ and $150 \mathrm{mM} \mathrm{NaCl}$ using L-glutamic acid $\gamma$-(7-amido-4-methylcoumarin) (L-GluAMC) from Sigma as the substrate. Rates were measured at $37^{\circ} \mathrm{C}$ by monitoring the release of AMC continuously (excitation at $380 \mathrm{~nm}$ and emission at $460 \mathrm{~nm}$ ). The fluorescence intensity of AMC was proportional to the concentration up to $2 \mu \mathrm{M}$, and inner filter effect corrections were not necessary.

Cloning and Expression of K562N, K562Q, and Y403A HuGGT Variants. WT HuGGT and the K562N, K562Q, and Y403A variants were expressed by the same method described above using six-well plates and $500 \mu \mathrm{g}$ of plasmid and $2.5 \mu \mathrm{L}$ of lipofectamine per well. Two controls were included: (1) cells treated with lipofectamine and no DNA and (2) untreated cells. The cells were lysed in $250 \mu \mathrm{L}$ of lysis buffer, and the cell lysates were clarified by centrifugation at $10,000 \mathrm{~g}$ for $10 \mathrm{~min}$. Protein concentrations were measured using a Bradford assay (Bio-Rad, Inc.). Activity was measured using the L-Glu-AMC substrate with or without an acceptor substrate and 1:20 dilutions of the enzyme (enzyme diluted in assay buffer). Reaction mixtures contained $5 \mu \mathrm{L}$ of the diluted enzyme and $95 \mu \mathrm{L}$ of the substrates in assay buffer [50 mM HEPES ( $\mathrm{pH} 8.0$ ) and $150 \mathrm{mM} \mathrm{NaCl}$. $\mathrm{IC}_{50}$ values were measured using the same lysates.

Modeled Substrates and Inhibitors of HuGGT and CapD. It is well-known that GGTs use glutathione (GSH), a $\gamma$-L-Glu-L-Cys-Gly tripeptide, as a donor substrate. However, CapD has not been shown to hydrolyze $\gamma$-glutamyl- $p$ nitroanilide ${ }^{9}$ or glutathione. ${ }^{3}$ In B. anthracis, CapD binds and hydrolyzes a polymer of $\gamma$-D-glutamic acid, PDGA, which forms the capsule. To model PDGA bound to CapD, we constructed four derivatives of $\gamma$-D-Glu-D-Glu dipeptides in all four capping combinations (i.e., acetyl and/or $N$-methyl capping groups at the $\mathrm{N}$ - and $\mathrm{C}$-termini) and refined their complexes using $\mathrm{MD}$ simulations (see the Supporting Information). Figure 1 shows the glutathione (GSH) donor substrate, the HuGGT 
<smiles>[NH3+]C(CCC(=O)NC(CS)C(=O)NCC(=O)O)C(=O)[O-]</smiles>

Glutathione (GSH)
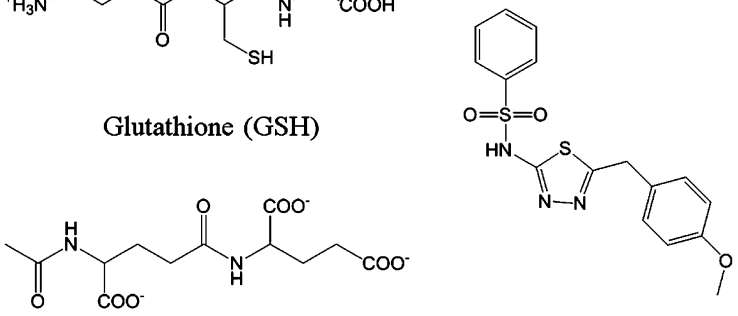

OU749

Acetylated di- $\gamma$-glutamic acid

Figure 1. Small molecule probes for GGT used in this study. The glutathione HuGGT substrate and B. anthracis CapD substrate mimic, acetylated di- $\gamma$-glutamic acid, are shown. The OU749 acceptor site inhibitor of HuGGT was described by King et al. ${ }^{34}$

acceptor site inhibitor, OU749, ${ }^{38}$ and the capped PDGA CapD substrate mimic.

Homology Modeling of HuGGT. The atomic structure of HuGGT was built from the crystal structure of EcGGT. Both the native structure and the $\gamma$-glutamyl-bound structure of EcGGT were used as templates (PDB entries 2DBU and 2DBW, respectively). ${ }^{20}$ The restraint-based comparative modeling program MODELER was used to construct the structural models of HuGGT in the native form and $\gamma$-glutamylbound complex. ${ }^{39}$ Residues 1-34 and 366-380 were excluded from the HuGGT model, as these are typically disordered in the structures of homologues. A set of 20 models from random generation of the starting structure was minimized, and the representative model was selected with the lowest value of MODELER objective function. The three-dimensional (3D) fold of the generated models was verified with PROSA II, ${ }^{40}$ and problematic regions were inspected via analysis of the PROSA II energy profile. The resulting models were further optimized with an iterative approach until no significant improvement was obtained. The stereo chemical quality and protein structure of the final models were validated by PROCHECK. ${ }^{41}$

Molecular Dynamics Simulations. Molecular dynamics (MD) simulations were conducted for modeled systems in explicit solvent using the AMBER 10 package and the Parm99SB force field. ${ }^{42}$ The solvated protein systems were subjected to energy minimization prior to $\mathrm{MD}$ simulations by first minimizing the water molecules while holding the solute frozen (1000 steps using the steepest descent algorithm), followed by 5000 steps of conjugate gradient minimization of the whole system to remove close contacts and to relax the system. Bond lengths involving hydrogen were constrained with SHAKE, and the time step for all MD simulations was set to 2 fs. A nonbonded cutoff of $10 \AA$ was used, and the nonbonded pair list was updated every 25 steps. Periodic boundary conditions were applied in simulations using the particle mesh Ewald (PME) method to calculate the long-range electrostatic interactions. The simulated system was first subjected to a gradual temperature increase from 0 to $300 \mathrm{~K}$ over $100 \mathrm{ps}$ and then equilibrated for $500 \mathrm{ps}$ at $300 \mathrm{~K}$, followed by production runs with a total length of 2 ns. Constant temperature and pressure ( $300 \mathrm{~K}$ and $1 \mathrm{~atm}$, respectively) were maintained using the Berendsen coupling algorithm with a time constant for heat-bath coupling of 0.2 ps. The resulting trajectories were analyzed using the PTRAJ module from AMBER. The rootmean-square deviations (rmsds) of the backbone were calculated from the trajectories at 1 ps intervals, with the initial structure as the reference. The root-mean-square positional fluctuations ( $\mathrm{rmsfs}$ ) were calculated on the basis of superposition of all $\mathrm{C}_{\alpha}$ atoms using the averaged structure of trajectories as the reference and compared to the experimental $B$ factors.

Ensemble Docking and Binding Free Energy Calculations. The AutoDock-based DOVIS program ${ }^{43}$ was used for docking the substrates to the modeled structure of HuGGT and CapD. Multiple conformations associated with the active site residues were obtained from $\mathrm{MD}$ simulations and used for all docking studies. The active site of the protein was defined by a grid of $70 \times 70 \times 70$ points with a grid spacing of $0.375 \AA$ centered at the catalytic residue Thr. The Lamarckian genetic algorithm (LGA) in AutoDock was applied with 50 runs, and the maximal number of energy evaluations was set to $2 \times 10^{6}$. Results differing by $<1.0 \AA$ in the positional rmsd of the substrate were clustered, and the one with the most favorable free energy of binding represented the final binding conformations. The optimal binding complexes were subjected to a stepwise energy minimization and MD simulations in explicit solvent as described above. The binding free energies were calculated using the MM-GBSA method as described previously. ${ }^{44}$ A set of 300 snapshots was extracted from trajectories of binding complexes at 10 ps intervals from the last 3 ns of each MD simulation. The molecular mechanics interaction energies were calculated with the SANDER module in the Amber 10 package. The entropy was estimated through normal-mode analysis using the NMODE module.

\section{RESULTS}

Kinetic Analysis of the Acceptor Substrate Preferences in CapD. Using the purified poly-D-glutamate (PDGA) capsule from the Ames strain of $B$. anthracis, we initially observed a faster rate of $B$. anthracis PDGA capsule degradation in the presence of cell culture medium versus buffer. Analysis of PDGA capsule degradation by agarose gel electrophoresis indicated that the transpeptidase activity was greater than the hydrolase activity. The components of the media were tested individually; some but not all amino acids were able to enhance capsule cleavage (Figure S1 of the Supporting Information). To quantify the rate of transpeptidation and to identify any unique amino acid acceptor substrate preferences of the bacterial GGT, CapD, that differ from those of rat GGT (and presumably HuGGT), the steady state kinetic parameters were measured for each amino acid by globally fitting three-dimensional data sets (Table 1). Both FRET donor and acceptor substrate concentrations were varied and yielded a set of parallel lines in a double-reciprocal plot consistent with a ping-pong bi-bi mechanism (binary complex) (Figure S2 of the Supporting Information).

From the three-dimensional kinetic analysis, we found that the $K_{\mathrm{ms} \text {,app }}$ values for the FRET donor substrate varied for each of the amino acid acceptor substrates. For a ping-pong mechanism, the binding of the donor substrate and formation of the covalent intermediate are expected to be the same regardless of the acceptor substrate. Thus, the variation likely arises from competitive inhibition by the acceptor substrate; this was previously suggested by Thompson and Meister in their studies of rat GGT. ${ }^{26}$

Comparison of the bimolecular rate constants, $k_{\mathrm{cat}} / K_{\mathrm{ma}}$ for each amino acid acceptor substrate showed the order of the CapD acceptor substrate preference: L-Cys > L-Glu > L-Gln > D-Glu = L-Met (Table 1). Of the acceptors tested, L-Cys yielded 
Table 1. Acceptor Substrate Preferences for CapD-MBP in $25 \mathrm{mM}$ HEPES (pH 7.4) and 0.1\% Tween 20 at $22 \pm 3{ }^{\circ} \mathrm{C}^{a}$

\begin{tabular}{|c|c|c|c|c|c|}
\hline acceptor substrate & $K_{\mathrm{ms,app}}(\mathrm{FRET}$ donor substrate) $(\mu \mathrm{M})$ & $K_{\mathrm{ma}}($ acceptor $)(\mu \mathrm{M})$ & $V_{\max }($ Units $/ \mathrm{mg})$ & $k_{\text {cat }}\left(\min ^{-1}\right)$ & $k_{\text {cat }} / K_{\mathrm{ma}}\left(\min ^{-1} \mu \mathrm{M}^{-1}\right)$ \\
\hline $\mathrm{H}_{2} \mathrm{O}$ & $4 \pm 2$ & - & $0.0019 \pm 0.0003$ & $0.19 \pm 0.03$ & - \\
\hline L-alanine & $6 \pm 2$ & $5000 \pm 1000$ & $0.011 \pm 0.002$ & $1.1 \pm 0.2$ & $0.00022 \pm 0.00006$ \\
\hline L-arginine & $50 \pm 10$ & $40000 \pm 10000$ & $0.07 \pm 0.02$ & $7 \pm 2$ & $0.00018 \pm 0.00006$ \\
\hline L-asparagine & $9 \pm 3$ & $15000 \pm 5000$ & $0.035 \pm 0.008$ & $3.5 \pm 0.8$ & $0.00023 \pm 0.00009$ \\
\hline L-aspartate & $7 \pm 3$ & $300 \pm 100$ & $0.0044 \pm 0.0008$ & $0.44 \pm 0.08$ & $0.0015 \pm 0.0006$ \\
\hline L-cysteine & $12 \pm 2$ & $170 \pm 20$ & $0.023 \pm 0.002$ & $2.3 \pm 0.2$ & $0.014 \pm 0.002$ \\
\hline D-glutamate & $8 \pm 3$ & $150 \pm 40$ & $0.006 \pm 0.001$ & $0.6 \pm 0.1$ & $0.004 \pm 0.001$ \\
\hline L-glutamate & $22 \pm 7$ & $400 \pm 100$ & $0.028 \pm 0.006$ & $2.8 \pm 0.6$ & $0.007 \pm 0.002$ \\
\hline L-glutamine & $14 \pm 9$ & $400 \pm 300$ & $0.02 \pm 0.01$ & $2 \pm 1$ & $0.005 \pm 0.005$ \\
\hline glycine & $13 \pm 11$ & $5000 \pm 4000$ & $0.008 \pm 0.004$ & $0.8 \pm 0.4$ & $0.0002 \pm 0.0002$ \\
\hline L-histidine & $3 \pm 2$ & $210 \pm 150$ & $0.006 \pm 0.001$ & $0.6 \pm 0.1$ & $0.003 \pm 0.002$ \\
\hline L-isoleucine & $7 \pm 2$ & $25000 \pm 6000$ & $0.019 \pm 0.003$ & $1.9 \pm 0.3$ & $0.00008 \pm 0.00002$ \\
\hline L-leucine & $8 \pm 4$ & $600 \pm 200$ & $0.016 \pm 0.004$ & $1.6 \pm 0.4$ & $0.003 \pm 0.001$ \\
\hline L-lysine & $19 \pm 9$ & $800 \pm 500$ & $0.010 \pm 0.003$ & $1.0 \pm 0.3$ & $0.0013 \pm 0.0009$ \\
\hline L-methionine & $2 \pm 1$ & $80 \pm 30$ & $0.0029 \pm 0.0005$ & $0.3 \pm 0.05$ & $0.004 \pm 0.002$ \\
\hline L-phenylalanine & $8 \pm 4$ & $1500 \pm 500$ & $0.015 \pm 0.004$ & $1.5 \pm 0.4$ & $0.0010 \pm 0.0004$ \\
\hline L-proline & $\mathrm{NA}^{b}$ & & & & \\
\hline L-serine & $7.8 \pm 0.7$ & $990 \pm 80$ & $0.021 \pm 0.001$ & $2.1 \pm 0.1$ & $0.0021 \pm 0.0002$ \\
\hline L-threonine & $6 \pm 4$ & $1400 \pm 900$ & $0.010 \pm 0.003$ & $1.0 \pm 0.3$ & $0.0007 \pm 0.0005$ \\
\hline L-tryptophan & $13 \pm 6$ & $1500 \pm 500$ & $0.013 \pm 0.003$ & $1.3 \pm 0.2$ & $0.0009 \pm 0.0003$ \\
\hline L-valine & $20 \pm 10$ & $32000 \pm 17000$ & $0.03 \pm 0.01$ & $3.0 \pm 1.0$ & $0.00009 \pm 0.00006$ \\
\hline
\end{tabular}

$a_{\mathrm{L}-T y r o s i n e}$ was not tested because of its poor solubility at neutral $\mathrm{pH}$. Steady state kinetic parameters for CapD without MBP were measured using $\mathrm{L}$-Cys $\left[K_{\mathrm{ms}, \mathrm{app}}(\right.$ substrate $)=5 \pm 2 \mu \mathrm{M}, K_{\mathrm{ma}}=220 \pm 60 \mu \mathrm{M}, V_{\max }=0.024 \pm 0.004$ Units $/ \mathrm{mg}$, and $\left.k_{\text {cat }}=1.3 \pm 0.2 \mathrm{~min}^{-1}\right]$ and were found to be comparable to those measured for CapD-MBP. ${ }^{b}$ No significant increase in activity was observed with $50 \mathrm{mM}$ L-proline. L-Proline does not have a primary amine that can function as an acceptor in the reaction.

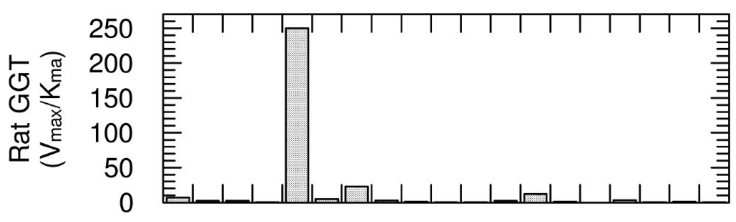

A R N D C EQGH I L KM F P S T V

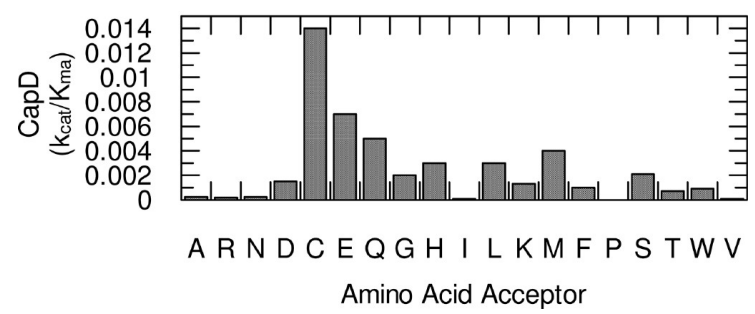

Figure 2. Comparison of acceptor substrate preferences for rat GGT (published values from ref 26) and B. anthracis CapD (from Table 1). Both GGT enzymes prefer L-Cys as their acceptor; however, CapD, unlike rat GGT, can also utilize L-Glu and other amino acids as acceptor substrates.

the highest $k_{\text {cat }} / K_{\mathrm{ma}}$ and L-Met had the lowest $K_{\mathrm{ma}}$. CapD, therefore, had an acceptor preference similar to that of rat GGT (which is 79\% identical to the HuGGT sequence) and has been reported to have the same acceptor substrate preference for L-Cys and the highest affinity for L-Met (Figure 2). ${ }^{13,26}$ Rat GGT prefers the L-Cys acceptor substrate most likely because of its similarity to its donor substrate (glutathione) and product (cysteinyl-glycine). However, for CapD, this result was unexpected because glutathione has not been shown to be a donor substrate. The common acceptor preference observed between CapD and rat GGT suggests that, although their donor substrate preferences are distinctly different, CapD and rat GGT may share similar binding interactions within their acceptor sites.
Further examination of the CapD acceptor substrate preferences showed that CapD could utilize a broader range of acceptor substrates than rat GGT (Figure 2). Because CapD hydrolyzes an unusual poly-D-glutamate donor substrate, ${ }^{3}$ we tested the negatively charged D-Glu amino acid as an acceptor substrate in transpeptidation. A 2.7-fold lower $K_{\mathrm{ma}}$ was found for D-Glu compared to that for L-Glu. However, the turnover number, $k_{\text {cat }}$ with D-Glu was 4.7-fold lower than that with L-Glu. Overall, the specificity constant, $k_{\mathrm{cat}} / K_{\mathrm{ma}}$ for L-Glu was 1.8-fold higher than for D-Glu (Table 1), indicating that the acceptor site of CapD does not strongly prefer D-amino acids over L-amino acids. Rat GGT and EcGGT have strict stereospecificity for L-amino acid acceptors over D-amino acids. The lack of stereospecificity in CapD demonstrates that the acceptor site of CapD, while similar in some respects, is distinctly different from that of HuGGT. To understand the lack of stereospecificity and the acceptor substrate preference for negatively charged amino acids, we utilized both sitedirected mutagenesis and molecular modeling of the $\mathrm{D}$ - and L-amino acid acceptor substrates.

Site-Directed Mutagenesis Studies of the CapD Acceptor Site. Examination of the putative acceptor substrate site in CapD suggested that Arg-432 and Arg-520 may both be involved in binding the acceptor substrate (Figure $3 \mathrm{~A}$ and Figures S3 and S4 of the Supporting Information). For CapD, the L-Arg acceptor substrate yielded the highest $k_{\text {cat }}$. The higher turnover number may be due to faster product release, as the $K_{\text {ma }}$ of this acceptor $[40 \pm 10 \mathrm{mM}$ (Table 1$\left.)\right]$ was the highest of the amino acids tested. Docking indicated that the poor binding of this acceptor may be due to Arg-432, which is predicted to be within the putative acceptor site (Figure 3A). This result was similarly observed for EcGGT, ${ }^{19}$ suggesting that the two bacterial GGTs share a similar residue within their acceptor substrate sites (Figure 3B). EcGGT also has an 
A

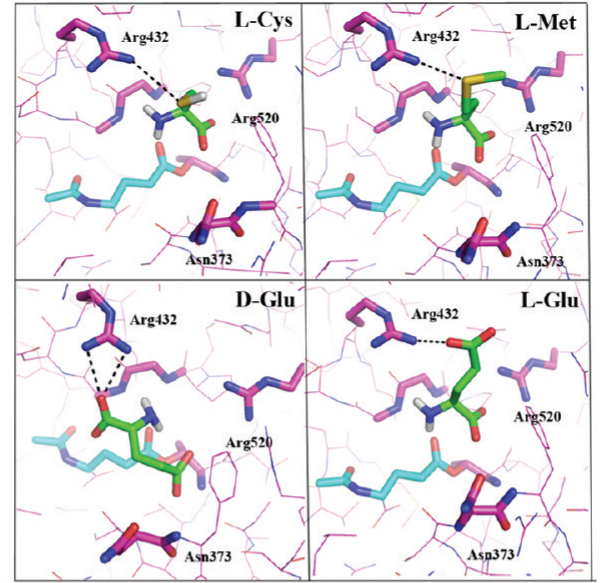

B

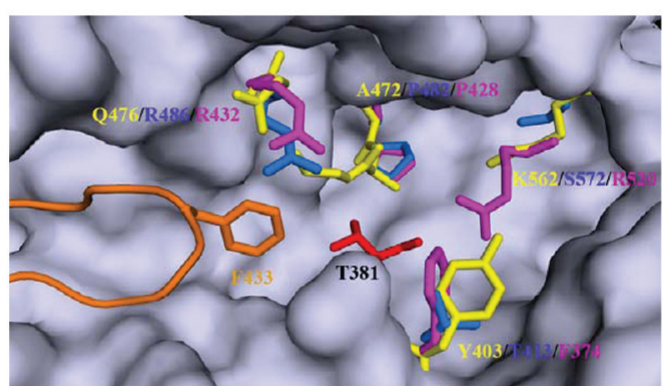

\begin{tabular}{|c|c|c|c|c|c|c|}
\hline & 373 & 392 & (1oop) & 428 & 498 & 520 \\
\hline & 1 & 1 & 1 & 1 & 1 & 1 \\
\hline . anthracis & NFFG. & QNFG. & ---- & . GIGSPGGNRI. & . FGGVQ. & DGRRNG \\
\hline subtilis & LFG. & TDFD. & . & TVGSPGGATI. & . IGNVQ. & .DSSRNG \\
\hline .aureus & IIYG. & DGFD. & --- & .TVGAPGAISI. & . YIGDV. & .DDTREG \\
\hline E.coli & TTFG. & .DDFS. & .VYGLV. & . .VTGSPGGSRI. & .MGSTQ. & . . DPRSVD \\
\hline g.coli 0157 & GYFG. & .DDFS. & .VYGLV. & . .VTGSPGGSLI. & .MGSTQ. & . .DPRSVD \\
\hline I.pylori & ASYG. & DDFS. & .LYGLV. & . .VVGSPGGSRI. & . MGDVN. & . DPRKEF \\
\hline tularensis & YSYG. & .DDFA. & . VFGLV. & . .ATGSPGGSRI. & . -MGAA. & . DPRRAS \\
\hline Y.pestis & GRFG. & DDFT. & . LYGLV. & . .VLGSPGGSRI. & . -WGAA. & . . DVRRPA \\
\hline 3.mallei & GYFG. & DDFS. & .MFGVV. & . .VIGTPGGSRI. & $\ldots$-SGDI. & . .DPRGRG \\
\hline ouse & LYFG & .DDFS. & . QF'RVA. & . .VVGASGGTQI. & . FIAVV. & . .DSRKGG \\
\hline at & LYFG & .DDFS. & . QFGVA. & . . VVGASGGTQI. & . FIAVV. & . .DSRKGG \\
\hline ig & LYFG & DDFS & . QFGVR. & .VVGASGGTQI . & . FIGVV. & . .DSRKGG \\
\hline Iuman & LYFG. & .DDFS & .EFGVP. & . . VVGAAGGTQI. & . FIAVV. & . .DSRKGG \\
\hline & 1 & 1 & 1 & 11 & 1 & 1 \\
\hline & 403 & 423 & 433 & 476 & 542 & 562 \\
\hline
\end{tabular}

Figure 3. (A) Predicted binding models of preferred amino acid acceptors of CapD. (B) Structural and sequence alignment of the putative acceptor site of CapD with those of other GGTs. The numbering at the top corresponds to that of CapD (purple); the numbering at the bottom of the alignment corresponds to that of the human enzyme (yellow). The residues corresponding to EcGGT are colored blue.

arginine at the position homologous to Arg-432 in CapD, while in HuGGT, a neutral glutamine is present. We hypothesized that differences between the bacterial and human acceptor substrate preferences could arise from this charged residue.

To further validate our predicted acceptor site, we sought experimental evidence and characterized three CapD variants, N373A, R432A, and R520S (Table 2). The Asn-373 variant was selected because it is approximately $180^{\circ}$ from Arg- 432 . Analysis of the kinetic parameters of each of the variants allowed the orientation of the side chain of the acceptor amino acid in our models to be verified. To establish the orientation of the $\mathrm{COO}^{-}$group of the acceptor substrate, which could point toward either Arg-520 or Arg-432, we also selected the R520S variant. In HuGGT, the residue homologous to Arg-520 is conservatively substituted with Lys-562. Previously, no role for this positively charged residue had been established in catalysis.

Site-directed mutagenesis of Arg-520 to serine showed a $>95$-fold decrease in $k_{\text {cat }}$ (hydrolysis) consistent with a role for Arg-520 in catalysis and/or product release (Table 2). The activity of the R520S variant was not stimulated by the addition of an amino acid acceptor substrate, and no detectable activity for this variant could be measured (a 1400-fold decrease in $k_{\text {cat }}$ compared to that of WT CapD using L-Cys as the acceptor substrate). Thus, the role of Arg-520 may be to interact with the carboxylate of the amino acid acceptor substrate and function as an electrostatic catalyst.

Examination of a second variant, R432A, showed a smaller 6-fold decrease in $k_{\text {cat }}$ and no significant change in the apparent $K_{\mathrm{ms}}$ of the donor substrate when water functioned as the acceptor substrate. The 4.9 -fold decrease in $k_{\text {cat }} / K_{\mathrm{m}}$ for the hydrolase activity suggests a minor role for Arg-432 in catalysis. However, like the R520S variant, no significant stimulation of the activity was observed when the Gly, L-Asp, D-Glu, or L-Glu acceptor was present at $5 \mathrm{mM}$. Rather, a decrease in activity was observed when D-Glu, L-Glu, or L-Asp was added. Further examination of the R432A variant showed that L-Glu could now 
Table 2. Steady State Kinetic Parameters for CapD Acceptor Site Mutants

\begin{tabular}{|c|c|c|c|c|c|c|}
\hline enzyme & acceptor substrate & $K_{\mathrm{ms,app}}(\mathrm{FRET}$ donor substrate $)(\mu \mathrm{M})$ & $K_{\mathrm{ma}}($ acceptor $)(\mu \mathrm{M})$ & $V_{\max }($ Units/mg) & $k_{\text {cat }}\left(\min ^{-1}\right)$ & $k_{\text {cat }} / K_{\mathrm{ma}}\left(\min ^{-1} \mu \mathrm{M}^{-1}\right)$ \\
\hline \multirow[t]{8}{*}{ R432A } & $\mathrm{H}_{2} \mathrm{O}$ & $3.2 \pm 0.7$ & & $0.00031 \pm 0.00002$ & $0.031 \pm 0.002$ & \\
\hline & $\mathrm{H}_{2} \mathrm{O}$ & $-{ }^{a}$ & & $0.00031 \pm 0.00001$ & $0.031 \pm 0.001$ & \\
\hline & glycine & $10 \pm 10$ & $>20000$ & $>0.0010$ & $>0.10$ & \\
\hline & L-arginine & $10 \pm 6$ & $1500 \pm 1400$ & $0.0024 \pm 0.0008$ & $0.24 \pm 0.08$ & $0.0002 \pm 0.0002$ \\
\hline & L-aspartate & $-^{a}$ & $>5000$ & $\geq 0.00007$ & $\geq 0.007$ & \\
\hline & L-cysteine & $-^{a}$ & $>5000$ & $\geq 0.0005$ & $\geq 0.05$ & \\
\hline & D-glutamate & $-^{a}$ & $>5000$ & $\geq 0.00007$ & $\geq 0.007$ & \\
\hline & L-glutamate & $-{ }^{a}$ & $>5000$ & $\geq 0.0001$ & $\geq 0.01$ & \\
\hline \multirow[t]{4}{*}{ N373A } & $\mathrm{H}_{2} \mathrm{O}$ & $3 \pm 1$ & & $0.00050 \pm 0.00008$ & $0.050 \pm 0.008$ & \\
\hline & L-cysteine & $10 \pm 3$ & $500 \pm 100$ & $0.008 \pm 0.001$ & $0.8 \pm 0.1$ & $0.0016 \pm 0.0004$ \\
\hline & D-glutamate & $5 \pm 2$ & $60 \pm 40$ & $0.006 \pm 0.002$ & $0.6 \pm 0.2$ & $0.010 \pm 0.007$ \\
\hline & L-glutamate & $15 \pm 8$ & $120 \pm 70$ & $0.015 \pm 0.006$ & $1.5 \pm 0.6$ & $0.013 \pm 0.009$ \\
\hline \multirow[t]{2}{*}{ R520S } & $\mathrm{H}_{2} \mathrm{O}$ & $-{ }^{b}$ & & $\leq 0.00002$ & & \\
\hline & L-glutamate & $-{ }^{a}$ & $>5000$ & $\leq 0.00002$ & & \\
\hline
\end{tabular}

act as a competitive inhibitor with a $K_{\mathrm{i}}$ of $0.5 \pm 0.3 \mathrm{mM}$. The $K_{\mathrm{ma}}$ for L-Arg with the R432A variant decreased dramatically from 40 to $1.5 \mathrm{mM}$ [26-fold (Table 2)], corroborating the orientation of the acceptor substrate side chain toward R432. The $k_{\text {cat }}$ correspondingly decreased 29-fold, consistent with a slower rate of product release. Thus, the role of Arg-432 appears to be primarily in binding the $\mathrm{R}$ group of the L-amino acid acceptor substrates.

Conversely, D-amino acid acceptor substrates appear to employ Arg-432 in binding the $\alpha$-carboxylate, as opposed to Arg-520. The two alternate binding modes for $\mathrm{D}-$ and $\mathrm{L}^{-}$ acceptor substrates are shown in Figure 3. The two arginines could account for the lack of stereospecificity in CapD. EcGGT and rat GGT have strict stereospecificity for L-acceptor substrates and do not have both arginines (Figure 3B). Consistent with our models, the R432A variant was unable to utilize D-Glu or L-Glu as an acceptor substrate (Table 2). The sulfur atom of Met is known to form hydrogen bonds with $\mathrm{NH}$ groups. ${ }^{45-47}$ A hydrogen bond between the Met sulfur and Arg432 side chain may in part account for the tight binding of this acceptor (Figure 3).

A third variant, N373A, was examined. Asn-373 was also predicted to be near the putative acceptor substrate site (Figure 3). However, the N373A variant showed an only 2.9-fold decrease in $k_{\text {cat }}$ when water functioned as the acceptor. The $K_{\text {ma }}$ of L-Cys was also 2.9-fold higher than the $K_{\mathrm{ma}}$ of the L-Cys measured for WT CapD and was not significantly different, suggesting that the acceptor side chain is not oriented toward this residue. The measured $K_{\mathrm{ma}}$ values for D-Glu and L-Glu were also comparable to those of the wild type, suggesting that the Asn-373 side chain does not play a significant role in binding either stereoisomer.

Structural Properties of Human GGT and CapD. To improve our understanding of the structural basis of the acceptor preference associated with HuGGT and CapD, we applied computational approaches to probe the binding specificities of the substrate and inhibitors in the donor and acceptor sites. Because the structure of HuGGT is not available, we modeled it on the basis of homologous bacterial GGT. In addition to HuGGT, the structure of rat GGT was also modeled, which possesses an active site and ternary structure identical to those of HuGGT (Figure S5 of the Supporting Information). HuGGT shares the highest level of sequence identity ( $31 \%$ ) with EcGGT and levels of $20-28 \%$ with other bacterial GGTs from H. pylori, Bacillus subtilis (BsGGT), and
B. anthracis. The modeled structure of HuGGT showed an overall rmsd for $\mathrm{C}_{\alpha}$ atoms of $0.36 \AA$ with respect to the homologue, EcGGT, and values of $0.83,1.19$, and $1.29 \AA$ compared to the experimental structures of HpGGT, BsGGT, and CapD, respectively.

Structural analysis revealed that the same 3D fold is shared between human and bacterial GGTs (Figure 4). The most varied regions are located on the large $40 \mathrm{kDa}$ subunit and surface loops at the terminal end, while the catalytic binding site is mainly formed by the small $20 \mathrm{kDa}$ subunit, including the catalytic residue Thr-381 and the conserved oxyanion hole motif of Gly-483 and Gly-484 in HuGGT. Compared to CapD, the most distinct structural difference is the fact that HuGGT contains a substrate binding loop at the donor site (residues 427-438), whereas such a loop is absent in B. anthracis CapD as well the GGT in B. subtilis and S. aureus. ${ }^{48}$ Interestingly, another B. subtilis GGT containing a donor substrate binding loop has also been identified. ${ }^{49}$ The substrate-binding loop is found in many other bacterial GGTs and plays an important role in substrate recognition. ${ }^{22}$ The lack of the loop in CapD is strongly associated with the substrate and inhibitor binding specificities, as shown in the following modeling studies. Another notable difference between HuGGT and bacterial GGTs is that HuGGT possesses a "gating residue" Phe-433 within the substrate binding loop, while it is typically a tyrosine in bacterial GGTs.

Closer examination of the HuGGT and CapD structures suggested that the putative acceptor binding site is located in the region along the groove of the donor binding site (Figure 4). A large pocket is found in the proximity of the catalytic residue and $\gamma$-glutamyl intermediate that possibly accommodates various acceptor amino acids for the transpeptidation reaction. In contrast to the conserved donor site, the acceptor site residues varied greatly between human and bacterial GGTs. The binding pocket in HuGGT is formed by a number of polar residues such as Asp-46, Asn-79, His-81, Ser-82, Gln-476, Tyr-403, and Lys-562. The residues equivalent to Gln-476 and Lys-562 are substituted with Arg-432 and Arg-520, respectively, in CapD; these residues were shown to be involved in acceptor binding in our site-directed mutagenesis study. Residues Arg432 and Gln-476 are located on top of the donor-binding pocket and are predicted to form hydrogen bond interactions with the acceptor substrate (Figure 3). On the other hand, Arg-520 and Lys-562 point toward the acceptor site from the 

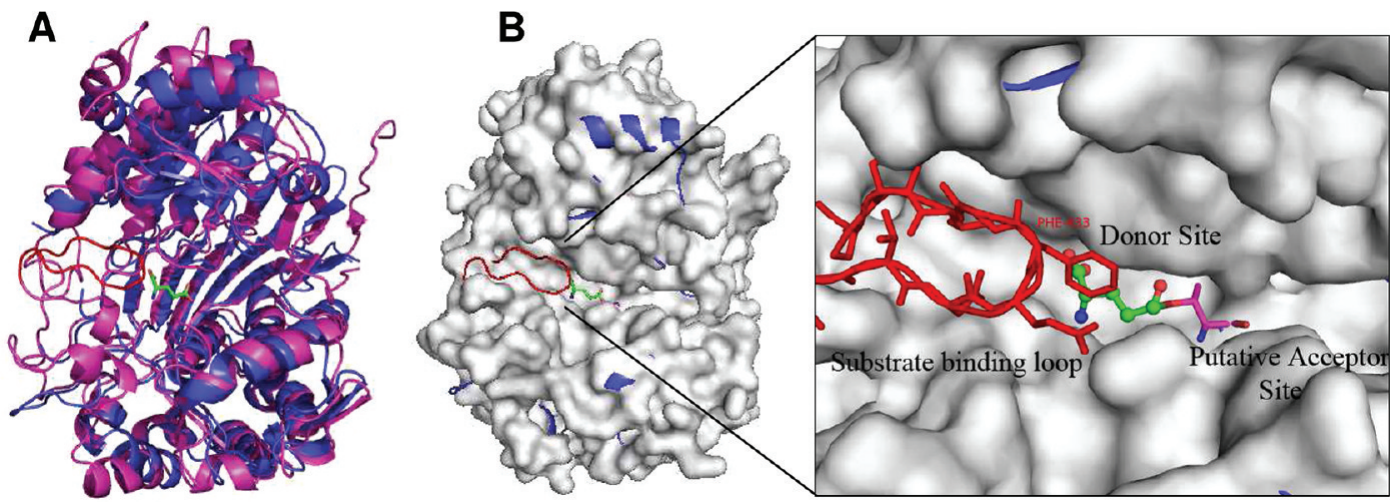

Figure 4. (A) Structural model of HuGGT (magenta ribbon and red loop) in comparison with CapD (blue ribbon, PDB entry 3 G9K). ${ }^{25}$ (B) HuGGT illustrated in surface representation (white). The HuGGT substrate-binding loop (red), nucleophilic residue T381 (magenta), and docked glutamyl group (green) are shown as sticks. The CapD structure is shown as ribbons (blue).

C-terminal loop of the small subunit. This residue appears to be highly flexible as two conformations were found in the X-ray crystal structure of $\mathrm{CapD},{ }^{25}$ and various conformations were seen in other bacterial GGTs. The motion of the C-terminal loop in HpGGT and the functional role in substrate binding have been reported recently. ${ }^{23}$ Notably, both HuGGT and CapD possess an aromatic residue (Phe-374 and Tyr-403, respectively) located in the front of the acceptor binding pocket, while a smaller residue is found in EcGGT (Thr-413) and HpGGT (Ser-402) with a rather open putative acceptor site. It is likely that this residue plays a functional role similar to that of the gating residue in the donor substrate-binding loop, shielding the acceptor substrate or inhibitor from solvent.

Dynamics of the Acceptor Substrate Site Occur in Cooperation with the Donor Substrate Site. To investigate the dynamic properties of HuGGT and CapD, we performed MD simulations for the apo and $\gamma$-glutamyl-enzyme intermediate (acylated) complexes in explicit solvent. Figure 5 depicts the atomic positional fluctuations of residues $\left(\mathrm{C}_{\alpha}\right)$ for both systems calculated from 2 ns of $\mathrm{MD}$ simulations. The calculated $B$ factors were generally in agreement with the experimentally measured data from the glutamyl-enzyme intermediate of EcGGT. ${ }^{20}$ Larger atomic fluctuations were mainly observed in loop regions on the protein surface and termini of two subunits (except for the N-terminal Thr-381 in HuGGT and Thr-352 in CapD). Both the donor and acceptor sites of HuGGT and CapD exhibited a higher level of conformational flexibility in the apo form, and the magnitude of the fluctuations decreased in the glutamyl-enzyme intermediate complex. The putative acceptor site appears to be more flexible than the donor site. Analysis showed that a well-formed hydrogen bonding network rigidifies the donor substrate binding site, while the acceptor site residues remain flexible. Interestingly, the presence of the $\gamma$-glutamyl moiety did not affect the CapD acceptor substrate binding site significantly, implying that the effect of the donor substrate binding on the acceptor site is different from that of HuGGT. It is worth noting that two small loops of HuGGT at the acceptor site, L2 (residues 459-464) and L3 (residues 549553), were found to exhibit large motions. In CapD, helix $\alpha 5$ (residues 167-179) in the back of the acceptor binding site is attenuated upon acylation of the enzyme.

Substrate binding loop L1 (residues 423-438) of HuGGT exhibited large fluctuations in the apo state as expected. The result is consistent with that observed in the apo structure of
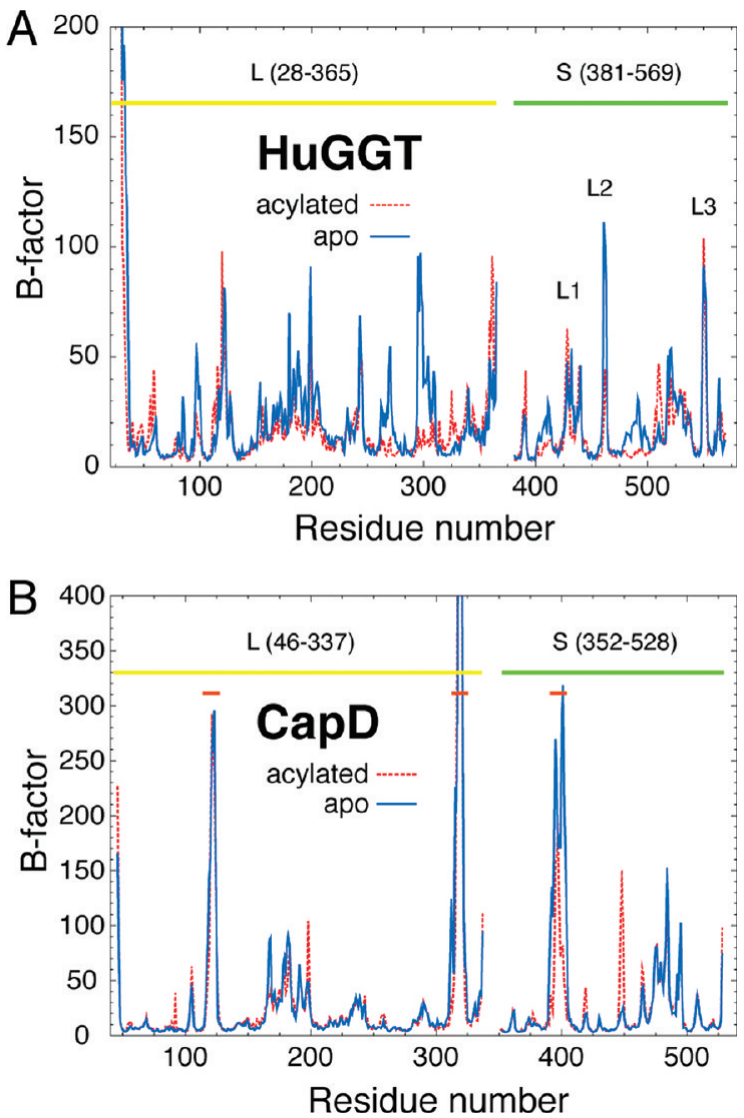

Figure 5. $B$ factors of $C_{\alpha}$ atoms computed from MD simulations of HuGGT (A) and CapD (B). Results for both apo (blue solid line) and acylated (red dashed line) forms are presented. Yellow and green horizontal bars atop each plot show the boundaries of the large $(\mathrm{L})$ and small (S) domains. In the acylated enzymes, the $B$ factors of the acyl $\mathrm{C}_{\alpha}$ atoms precede those of the catalytic threonine residues (T381 in HuGGT and T352 in CapD). The regions of high flexibility in CapD that are labeled with red bars correspond to modeled loops the density of which is not defined in the X-ray crystallographic structure (PDB entry 3G9K).

EcGGT and HpGGT. However, the $\gamma$-glutamyl moiety did not significantly reduce the flexibility of the L1 loop in HuGGT. Analysis showed that the gating residue, Tyr-444 in EcGGT and Tyr-433 in HpGGT, within the loop forms a hydrogen bond with the amide of the glutamyl moiety, whereas HuGGT 
A

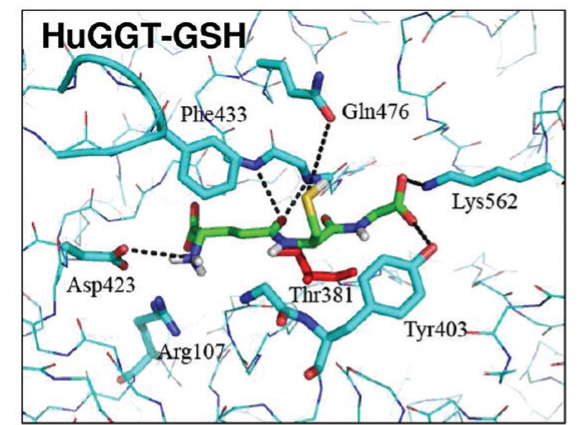

C

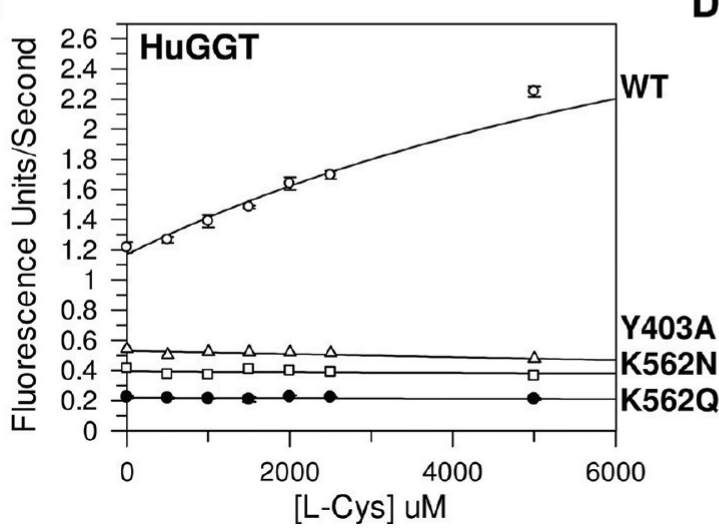

B
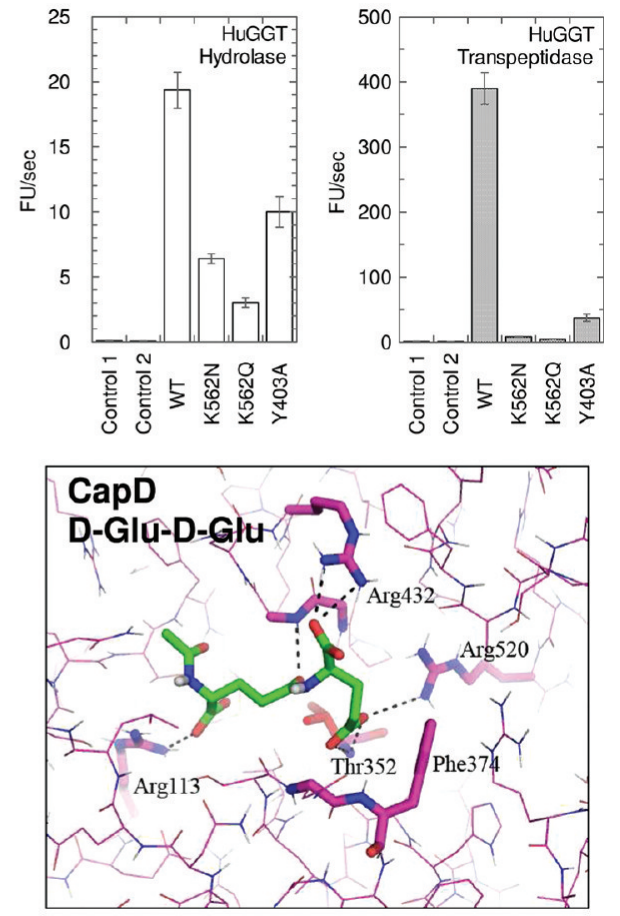

Figure 6. (A) Binding interactions of GSH in the active site of HuGGT. Residues involved in protein-ligand interactions are shown as sticks (cyan and red). For the sake of clarity, hydrogen atoms of the ligand have been omitted. Pairs of atoms involved in hydrogen bonding are connected with dashed lines. (B) Relative activity of WT HuGGT and the K562N, K562Q and Y403A variants with or without 40 mM glycyl-glycine. (C) No increase in activity was observed with increasing concentrations of the L-Cys acceptor substrate for the K562N, K562Q and Y403A variants. (D) Binding interactions of acetylated di- $\gamma$-glutamic acid (green) with CapD (magenta).

containing a Phe-433 gating residue lacks such a stabilizing binding interaction. As a result, the substrate binding loop of HuGGT in the substrate-bound complex exhibited larger fluctuations than EcGGT and HpGGT. The conformational change of the substrate binding loop, from open to closed, is thought to facilitate substrate recognition and catalysis. Therefore, the dynamics and higher flexibility of the substrate binding loop of HuGGT are likely associated with its higher transpeptidase activity (faster product release).

Differences in Donor Substrate Binding Specificities of HuGGT and CapD. GGTs that lack the donor substrate binding loop have not been shown to utilize glutathione as a substrate. The absence of this loop suggests a loss of key interactions with the GSH substrate of HuGGT and its glutamine analogue inhibitors such as acivicin, which inhibits CapD poorly $\left(\mathrm{IC}_{50}>20 \mathrm{mM}\right)$. To understand why $\mathrm{GSH}$ does not function as a substrate of $\mathrm{CapD}$, we investigated the binding specificity of GSH with HuGGT. Figure 6A shows the predicted complex of GSH bound in the active site of HuGGT. As expected, GSH adopted a binding mode similar to that observed in the experimental structure of the HpGGT T380A variant in complex with $S$-(nitrobenzyl)glutathione. ${ }^{22}$ Importantly, GSH interacts with both donor and acceptor binding sites of HuGGT. The $\gamma$-glutamyl moiety of GSH was buried deep in the pocket of the donor substrate site and formed an extensive hydrogen bonding network with residues Arg-103, Asn-401, Glu-419, Asp-423, Ser-451, and Ser-452. These residues are highly conserved among GGTs that contain the donor substrate binding loop, implying their primary functional role in donor substrate recognition. Moreover, reported mutational studies of HuGGT have supported the involvement of Asp-423, Ser-451, and Ser-452 in donor substrate binding. ${ }^{29,50}$ Asp-423 in HuGGT formed H-bond and electrostatic interactions with the $\alpha$-amino group of the $\gamma$-glutamyl moiety. In contrast, Asp-423 in CapD is substituted with an asparagine. The loss of this important interaction, together with the lack of a substrate binding loop, likely explains why GSH does not stably bind within the donor substrate site or function as a substrate in CapD.

Further analysis of the GSH binding mode indicated that the Cys-Gly leaving group pointed toward the putative acceptor site of HuGGT and formed two interesting interactions (Figure 6A). One potential hydrogen bond was found between the side chain of the Cys acceptor substrate and Asn-476, similar to the predicted binding model of acceptor L-Cys bound in CapD, as well as the structure of the HpGGT T380A variant bound to a glutathione analogue. ${ }^{22}$ Another interesting interaction was observed with residues Lys-562 and Tyr-403, which formed hydrogen bonds and strong charge-charge electrostatic interactions with the glycyl moiety of GSH. In contrast, residues Thr-413 and Ser-572 occupied these positions in EcGGT and did not participate in any interactions with the glycyl moiety in the modeled GSH-EcGGT complex. MD simulations showed that the Cys-Gly leaving group of GSH in HuGGT was stabilized because of well-formed interactions with residues Lys-562 and Tyr-403. This may benefit the nucleophilic attack in the transpeptidation reaction involving glycyl dipeptides and also account for the higher transpeptidase activity ${ }^{16}$ compared to that of EcGGT. ${ }^{19}$ 
CapD, unlike other GGTs, utilizes a polymer of $\gamma$-D-glutamic acid as a substrate. To probe the substrate binding specificities, we modeled four derivatives of $\gamma$-D-Glu-D-Glu dipeptides in all four capping combinations (i.e., acetyl and/or $N$-methyl capping groups at $\mathrm{N}$ - and C-termini, respectively) and docked them into the active site of CapD. Figure 6 shows that PDGA could interact with both the donor and acceptor substrate sites and bind between Arg-113 in the donor substrate site and Arg432 and Arg-520 in the putative acceptor substrate site. The substrate can bind in two different ways. Specifically, the substrate chain can swap its $\mathrm{N}$ - and C-termini. However, the conformer in which the Arg-113 side chain is closer to the $\mathrm{N}$-terminus appears to correspond to the productive conformation, as it satisfies the oxyanion hole constraint and has a favorable distance to the $\mathrm{O}_{\gamma}$ atom of the nucleophilic Thr-352 residue.

Site-Directed Mutagenesis Studies of the HuGGT Acceptor Site. To obtain experimental evidence for the predicted residues involved in substrate and inhibitor binding, we conducted site-directed mutagenesis studies of the HuGGT acceptor site. The His-tagged WT HuGGT was purified and assayed using the L-Glu-AMC substrate. For the hydrolysis reaction, a $K_{\mathrm{ms}}$ of $1.7 \pm 0.2 \mu \mathrm{M}$ was measured and agreed well with the $K_{\mathrm{ms}}$ previously measured by Han et al. ${ }^{18}\left(K_{\mathrm{ms}}=4 \mu \mathrm{M}\right)$ at $\mathrm{pH} 8.0\left(25^{\circ} \mathrm{C}\right)$ using the same substrate in the absence of an acceptor substrate. For the transpeptidase reaction, $K_{\mathrm{ms}}=16 \pm$ $9 \mu \mathrm{M}$ for the L-Glu-AMC donor substrate and $K_{\mathrm{ma}}=2200 \pm$ $1500 \mu \mathrm{M}$ for the L-Cys acceptor substrate. The $K_{\mathrm{ma}}$ of L-Cys for HuGGT was 6-fold higher than the $K_{\mathrm{ma}}$ of L-Cys reported for rat GGT $\left(K_{\mathrm{ma}}=340 \pm 60 \mu \mathrm{M}\right)^{26}$ at $\mathrm{pH} 8.0$ and $37^{\circ} \mathrm{C}$. The slightly higher $K_{\mathrm{ma}}$ of $\mathrm{L}-\mathrm{Cys}$ may be due to Ala-472, which is a serine in rat, pig, and mouse GGT.

To analyze the effects of the mutations, WT HuGGT and the $\mathrm{K} 562 \mathrm{~N}, \mathrm{~K} 562 \mathrm{Q}$ and $\mathrm{Y} 403 \mathrm{~A}$ variants were expressed in quadruplicate in six-well plates. Lysates of the COS-1 cells were assayed with the L-Glu-AMC donor substrate (Figure 6). Two controls were included: (1) no DNA and lipofectamine and (2) no DNA and no lipofectamine. Both controls showed $<0.4 \%$ of the activity measured for WT HuGGT, indicating that the GGT activity was predominantly that of the recombinantly expressed His-tagged GGT and not of endogenous GGT (a membrane-associated protein). Protein concentrations of the lysates were comparable for each well (data not shown). The steady state kinetic parameters with the L-Cys acceptor substrate were measured using the WT HuGGT lysate $\left(K_{\mathrm{ms}}=\right.$ $\left.4 \pm 1 \mu \mathrm{M} ; K_{\mathrm{ma}}=700 \pm 300 \mu \mathrm{M}\right)$ and were comparable to those measured using purified WT HuGGT. Mutation of Lys562 to asparagine or glutamine led to a 4- or 9-fold reduction in hydrolase activity, respectively. The loss of activity is consistent with a role for Lys-562 in electrostatic catalysis and/or product release. In the presence of the glycyl-glycine acceptor substrate, a 57- and 117-fold decreases in transpeptidase activity were observed for the $\mathrm{K} 562 \mathrm{~N}$ and $\mathrm{K} 562 \mathrm{Q}$ variants, respectively. The decreases in transpeptidase activity that were larger than those in hydrolase activity suggested a role for Lys-562 in neutralizing, binding, and/or orienting the acceptor substrate in the transpeptidation reaction.

Tyr-403 was predicted to be within hydrogen bonding distance of the glycyl group of the glutathione donor substrate (Figure 6). The Y403A variant showed approximately 3- and 14-fold decreases in hydrolase and transpeptidase activity (with the glycyl-glycine acceptor substrate), respectively, suggesting a minor role in catalysis of the hydrolysis reaction and a more significant role in the transpeptidation reaction.

Binding Interactions of the Acceptor Site Inhibitor OU749. To further probe the acceptor site, we explored the binding interactions of OU749, the first reported acceptor site inhibitor of a GGT. ${ }^{34}$ OU749 is a potent inhibitor of HuGGT; however, it does not inhibit CapD significantly $\left(\mathrm{IC}_{50}>1000\right.$ $\mu \mathrm{M})$, implying that residues involved in binding of OU749 in the acceptor site are not conserved in CapD. Figure 7A shows the effects of the $\mathrm{K} 562 \mathrm{~N}, \mathrm{~K} 562 \mathrm{Q}$, and $\mathrm{Y} 403 \mathrm{~A}$ variants on OU749 inhibition. All three variants significantly increased the $\mathrm{IC}_{50}$ from $30 \pm 10$ to $>2000 \mu \mathrm{M}$. Consistent with the experimental results, the predicted binding model of OU749 with HuGGT showed that Lys-562 pointed to the acceptor site and formed a hydrogen bond with the phenylcarboxylate group of OU749 (Figure 7B). On the other hand, Tyr-403 formed aromatic stacking interactions with the core thiadiazole group and shielded the molecule from solvent. Substitution of Lys562 with Gln or Asn or Tyr-403 with Ala disrupts the optimal binding interaction of OU749 in the acceptor site, and the calculated binding affinities were significantly decreased.

Interestingly, the phenyl group of OU749 was found to bind in an interior pocket of the putative acceptor site of HuGGT, which was not observed in the apo structure or the substrate binding complex. The pocket is located in the region behind the catalytic residue Thr-381 consisting mainly of hydrophobic residues such as Ala-471, Ala-472, Ala-542, and Val-544 (Figure 7). The two small flexible loops [L1 and L2 (Figure 5)] identified from MD simulations form the wall at the bottom of the pocket. In comparison, such a dynamic hydrophobic pocket was not observed in the putative acceptor site of CapD. Ser-427 and Pro-248 in CapD are positioned in the same site as Ala-471 and Ala-472. Docking and MD simulations showed that OU749 did not fit into the acceptor site of CapD; Ser-427 together with Arg-520 appeared to hinder OU749 binding.

A feature of OU749 inhibition is that the acceptor site inhibitor shows high selectivity toward HuGGT, but very poor inhibition of rat, mouse, and pig GGT. ${ }^{34}$ The species-specific inhibition by OU749 remains an interesting issue, as these GGTs are highly homologous, and the structural models of HuGGT and rat GGT do not reveal much difference in the donor or acceptor binding sites. Examination of the OU749HuGGT complex indicated that Ala-472 was positioned at the entrance of the hydrophobic pocket described above and could play a role in regulating access to this binding pocket. Ala-472 in HuGGT is replaced with a polar Ser in rat, mouse, and pig GGT. Substitution of Ala-472 with Ser would introduce a steric hindrance into the hydrophobic pocket. Moreover, the side chain could form a hydrogen bond with the lysine homologous to Lys-562. This interaction would be expected to block the binding of OU749. To confirm this, we modeled one HuGGT variant, $\mathrm{A} 472 \mathrm{~S}$, and performed $\mathrm{MD}$ simulations to probe the dynamic properties of the hydrophobic pocket. As shown in Figure 7, Ser-472 formed a stable H-bond with Lys-562, resulting in a closed conformation of the hydrophobic binding pocket in the acceptor site, whereas in WT HuGGT, Lys-562 exhibited high flexibility and the hydrophobic pocket remained in an open conformation. Therefore, the decreased inhibitory activities of OU749 against rat, mouse, and pig GGT are most likely due to the hydrogen-bonded conformation in the closed form, which would prevent OU749 binding. 
A

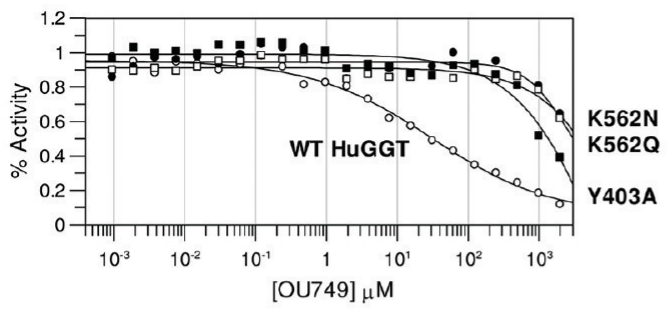

C

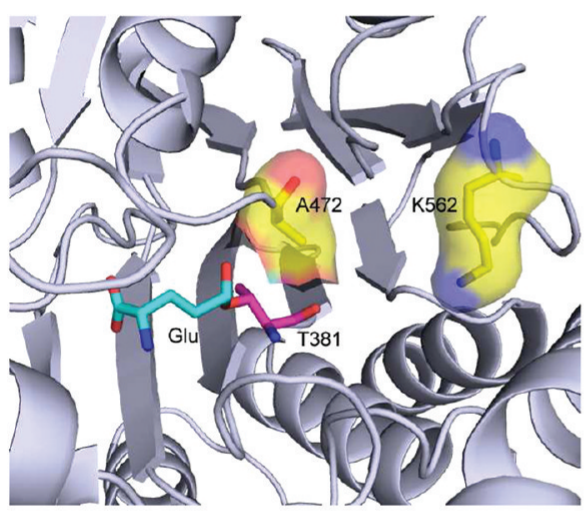

E

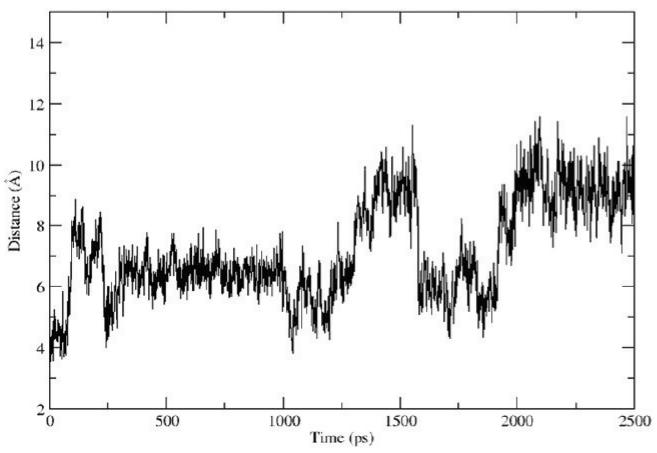

B

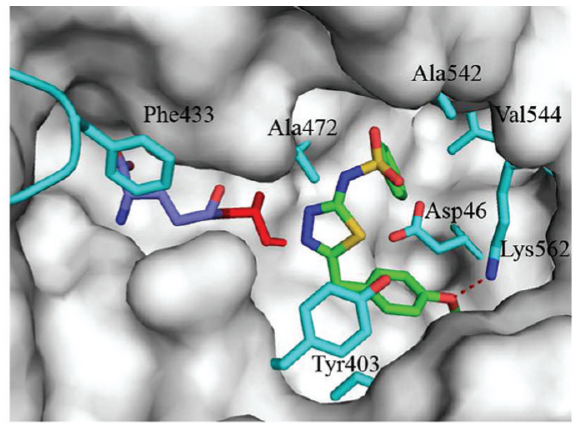

D

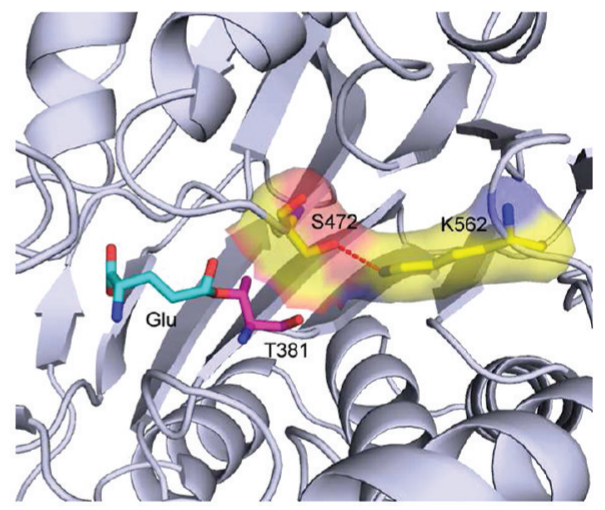

F

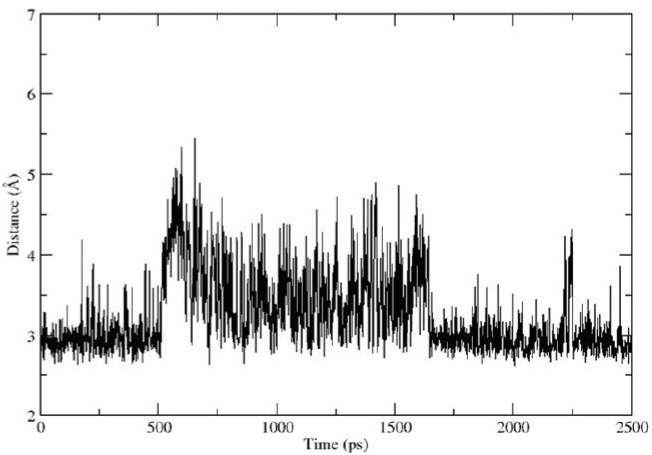

Figure 7. (A) $\mathrm{IC}_{50}$ values measured with WT HuGGT and the $\mathrm{K} 562 \mathrm{~N}, \mathrm{~K} 562 \mathrm{Q}$, and $\mathrm{Y} 403 \mathrm{~A}$ variants. (B) Binding interactions of OU749 with HuGGT (surface representation). The OU749 inhibitor and the active site residues of HuGGT are shown as sticks. (C) Open conformation of the acceptor site of HuGGT. The gating residues Lys-562 and Ala-472 are shown in a surface representation with the carbons colored yellow, oxygens red, and nitrogens blue. The glutamate substrate (cyan) and catalytic residue Thr-381 (magenta) are shown as sticks. (D) Closed conformation of the acceptor site observed in a model of an A472S mutant. The gating residue, Lys-562, can form an H-bond with Ser-472. (E) Plot of distances between residues Lys-562 and Ala-472 ( $\mathrm{C}_{\alpha}$ atoms) of HuGGT observed in the MD simulations. (F) Plot of distances between residues Lys-562 and Ser-472 ( $\mathrm{C}_{\alpha}$ atoms) of the A472S mutant during the MD simulations.

\section{DISCUSSION}

Within the acceptor site, we identified a positively charged residue (R520 in CapD and K562 in HuGGT) that is important for catalysis and acceptor substrate binding. Despite differences in the inhibitor and donor substrate preferences, both CapD and rat GGT (79\% identical to HuGGT) retain the same acceptor substrate preference (both prefer L-Cys) and have the lowest $K_{\mathrm{ma}}$ for L-Met (Figure 2 and Table 1). While the two enzymes can utilize the same acceptor substrate, they differ in their susceptibilities to the HuGGT acceptor site inhibitor, OU749, a species-specific inhibitor that does not significantly inhibit CapD. The two GGTs also differ in their acceptor substrate stereospecificity. Rat GGT has strict stereospecificity toward its acceptor substrates and can utilize only L-amino acids and dipeptides; ${ }^{13,26}$ CapD shows no significant preference for $\mathrm{L}-$ or D-amino acid acceptors (Table 1) and can accommodate both stereoisomers. Moreover, CapD comparably prefers negatively charged acceptor substrates such as D- or L-glutamate when compared to L-Cys. From the sitedirected mutagenesis and modeling described herein, it is clear that Arg-432 and Arg-520 play an important role in acceptor binding and likely allow the bacterial enzyme to utilize both D- and L-amino acid acceptors via two alternative binding modes (Figure 3): Arg-432 would be predicted to bind the $\alpha$-carboxylic acid of the amino acid for a D-isomer, while Arg-520 would bind it in the L-isomer. EcGGT has not been shown to 
utilize D-amino acid acceptors, ${ }^{27}$ and this may be due to its lack of an arginine equivalent to Arg-520 (EcGGT has a serine at this position). Correspondingly, HuGGT has a Gln at the position equivalent to Arg-432 of CapD and a Lys at the position equivalent to Arg-520 in CapD and has been shown to utilize only L-acceptor substrates. Several bacterial species (B. anthracis, F. tularensis, and Y. pestis) have GGTs that contain both arginines and may correspondingly lack acceptor substrate stereospecificity.

Mutation of Arg-520 led to the most significant decrease in $k_{\text {cat }}$ a 95 -fold decrease for hydrolysis and a 1400-fold decrease for transpeptidation. The modeling and kinetic analysis suggested a role for Arg-520 as an electrostatic catalyst. The Arg-432 and Arg-520 residues, as well as the transiently protonated N-terminal amino group of Thr-352 (which has been proposed to function as the general base and acid in Ntnamidohydrolases ${ }^{14,21}$ ), create a highly positively charged environment in the acceptor substrate site. Typical acceptor substrates would place a zwitterion near Thr-352. The neutral amine, however, is the likely nucleophile in the deacylation reaction (Scheme S1 of the Supporting Information). The proximity of Arg-520 to the $\alpha$-amino group of the acceptor substrate would likely make protonation of the amino group unfavorable and allow the neutral amine to function as a nucleophile in the transpeptidation reaction. Additionally, the electrostatic interaction between the acceptor $\mathrm{COO}^{-}$group and Arg-520 appears to be important for acceptor substrate binding. Han et al. ${ }^{33}$ speculated that a positively charged residue was present within the acceptor substrate site. On the basis of our kinetic analysis, modeling, and MD simulations, Lys-562 in HuGGT and Arg-520 in CapD appear to function as electrostatic catalysts and play an important role in acceptor substrate binding.

The transpeptidase activity has been proposed to serve an important physiological function and accounts for at least 50\% of the utilization of the $\gamma$-glutamyl donor. ${ }^{51}$ GGT present on the surface of some cell membranes is important for the breakdown of extracellular glutathione. The components are utilized or recombined; thus, both the hydrolase and transpeptidase activity appear to be important in GSH metabolism. In $B$. anthracis, both the transpeptidase and hydrolase activities function in anchoring and remodeling the capsule, respectively. From our results for CapD presented here and the previously published results for rat GGT and human GGT, ${ }^{13,30}$ it is apparent that the transpeptidase activity, regardless of the amino acid acceptor, is higher than the hydrolase activity (Table 1 and Figure 6). Mechanistically, it has been unclear why the transpeptidase activity is significantly greater than the hydrolase activity. Examination of the putative acceptor substrate site suggests that the negatively charged $\mathrm{COO}^{-}$ group of the acceptor substrate may neutralize the positive charge of the adjacent arginines, Arg-520 and Arg-432, and shield the $\alpha$-amine of Thr-352 from Arg-520. Water alone would not be sufficient to neutralize the charge of Arg-520. The shielding likely makes the protonation of the $\alpha$-amine of Thr352 more favorable during the catalytic cycle, accounting for the faster rate of transpeptidation versus hydrolysis (Scheme S1 of the Supporting Information).

The dynamics of the acceptor site and correlations with the donor substrate site are intriguing characteristics of GGT. Experimental studies revealed that the mechanism of inhibition of HuGGT by OU749 was uncompetitive relative to the $\gamma$-glutamyl substrate and suggested that the inhibitor preferentially interacted with the acylated $\gamma$-glutamyl enzyme intermediate over the free enzyme. ${ }^{34}$ The effect of the $\gamma$-glutamyl donor on the acceptor site appears to be subtle as the $\gamma$-glutamyl group did not form interactions with OU749 in the HuGGT-OU749 complex (Figure 7). Analysis of the dynamic behavior and conformational changes associated with the apo and $\gamma$-glutamyl enzyme intermediate in HuGGT indicated that two small loops, which form a hydrophobic pocket, exhibited high flexibility in the substrate-free form but were significantly stabilized in the glutamyl enzyme intermediate complex (Figure 5). The hydrophobic pocket in the acceptor site of HuGGT was observed in the substrate-bound complex but not apparent in the apo state. Therefore, a plausible explanation for the uncompetitive inhibition of OU749 is that the formation of the covalently bound glutamyl enzyme intermediate in the donor site facilitates rearrangement of the acceptor site and consequently favors the binding of acceptor site inhibitors (or acceptor substrates during transpeptidation).

Our overarching goal was to identify distinct differences between the bacterial and human GGT acceptor sites that may be useful in identifying species-specific inhibitors and/or broad spectrum antimicrobials of bacterial GGT. Here we demonstrated that the use of small molecule probes in combination with kinetic and computational analyses is an efficient approach to studying the underlying structural basis of substrate and inhibitor specificity. The characterization of the acceptor substrate site of HuGGT and B. anthracis CapD provides insight into the acceptor and donor substrate preferences associated with different species. More importantly, it has revealed a dynamic cooperation between the donor and acceptor sites in substrate recognition and catalysis. This synergy between the sites may be useful in the discovery of potent species-specific bisite inhibitors that bind to both the donor and acceptor sites.

\section{ASSOCIATED CONTENT}

\section{S Supporting Information}

Computational methods used for B. anthracis CapD, figures of the models of the $B$. anthracis CapD substrate and product complexes, a picture of the agarose gel from the $B$. anthracis capsule cleavage assay, and a proposed mechanism of CapDcatalyzed transpeptidation. This material is available free of charge via the Internet at http://pubs.acs.org.

\section{AUTHOR INFORMATION}

\section{Corresponding Author}

*X.H.: e-mail, xin.hu@nih.gov; telephone, (301) 217-1079; fax, (301) 217-5736. P.M.L.: e-mail, patricia.legler@nrl.navy.mil; telephone, (202) 404-6037; fax, (202) 404-8688.

\section{Present Address}

"NIH Chemical Genomics Center, National Human Genome Research Institute, National Institutes of Health, Bethesda, MD 20892.

\section{Author Contributions}

X.H. and P.M.L. contributed equally to this work.

\section{Funding}

This work was sponsored by Defense Threat and Reduction Agency Grants 2.10010.08.RD.B and CBM.THERB.02.11.RD.012 and the U.S. Department of Defense High Performance Computing Modernization Program, under the High Performance Computing Software Applications Institutes initiative. 


\section{Notes}

The authors declare no competing financial interest.

\section{ABBREVIATIONS}

CapD, capsule depolymerase; GGT, $\gamma$-glutamyl transpeptidase or $\gamma$-glutamyl transferase; HpGGT, H. pylori GGT; HuGGT, human GGT; EcGGT, E. coli GGT; FtuGGT, F. tularensis GGT; GSH, glutathione; MD, molecular dynamics; PDGA, poly-D-glutamic acid; WT, wild type.

\section{REFERENCES}

(1) Tate, S. S., and Meister, A. (1981) $\gamma$-Glutamyl transpeptidase: Catalytic, structural and functional aspects. Mol. Cell. Biochem. 39, 357-368.

(2) Taniguchi, N., and Ikeda, Y. (1998) $\gamma$-Glutamyl transpeptidase: Catalytic mechanism and gene expression. Adv. Enzymol. Relat. Areas Mol. Biol. 72, 239-278.

(3) Candela, T., and Fouet, A. (2005) Bacillus anthracis CapD, belonging to the $\gamma$-glutamyltranspeptidase family, is required for the covalent anchoring of capsule to peptidoglycan. Mol. Microbiol. 57, $717-726$.

(4) Hanigan, M. H. (1995) Expression of $\gamma$-glutamyl transpeptidase provides tumor cells with a selective growth advantage at physiologic concentrations of cyst(e)ine. Carcinogenesis 16, 181-185.

(5) Godwin, A. K., Meister, A., O'Dwyer, P. J., Huang, C. S., Hamilton, T. C., and Anderson, M. E. (1992) High resistance to cisplatin in human ovarian cancer cell lines is associated with marked increase of glutathione synthesis. Proc. Natl. Acad. Sci. U.S.A. 89, 3070-3074.

(6) Owen, A. D., Schapira, A. H., Jenner, P., and Marsden, C. D. (1996) Oxidative stress and Parkinson's disease. Ann. N.Y. Acad. Sci. 786, 217-223.

(7) Chevalier, C., Thiberge, J. M., Ferrero, R. L., and Labigne, A. (1999) Essential role of Helicobacter pylori $\gamma$-glutamyltranspeptidase for the colonization of the gastric mucosa of mice. Mol. Microbiol. 31, $1359-1372$.

(8) Shibayama, K., Kamachi, K., Nagata, N., Yagi, T., Nada, T., Doi, Y., Shibata, N., Yokoyama, K., Yamane, K., Kato, H., Iinuma, Y., and Arakawa, Y. (2003) A novel apoptosis-inducing protein from Helicobacter pylori. Mol. Microbiol. 47, 443-451.

(9) Richter, S., Anderson, V. J., Garufi, G., Lu, L., Budzik, J. M., Joachimiak, A., He, C., Schneewind, O., and Missiakas, D. (2009) Capsule anchoring in Bacillus anthracis occurs by a transpeptidation reaction that is inhibited by capsidin. Mol. Microbiol. 71, 404-420.

(10) Joyce, J., Cook, J., Chabot, D., Hepler, R., Shoop, W., Xu, Q., Stambaugh, T., ste-Amezaga, M., Wang, S., Indrawati, L., Bruner, M., Friedlander, A., Keller, P., and Caulfield, M. (2006) Immunogenicity and protective efficacy of Bacillus anthracis poly- $\gamma$-D-glutamic acid capsule covalently coupled to a protein carrier using a novel triazinebased conjugation strategy. J. Biol. Chem. 281, 4831-4843.

(11) Alkhuder, K., Meibom, K. L., Dubail, I., Dupuis, M., and Charbit, A. (2009) Glutathione provides a source of cysteine essential for intracellular multiplication of Francisella tularensis. PLoS Pathog. 5, e1000284

(12) Folk, J. E. (1969) Mechanism of action of guinea pig liver transglutaminase. VI. Order of substrate addition. J. Biol. Chem. 244, 3707-3713.

(13) Allison, R. D. (1985) $\gamma$-Glutamyl transpeptidase: Kinetics and mechanism. Methods Enzymol. 113, 419-437.

(14) Castonguay, R., Lherbet, C., and Keillor, J. W. (2003) Kinetic studies of rat kidney $\gamma$-glutamyltranspeptidase deacylation reveal a general base-catalyzed mechanism. Biochemistry 42, 11504-11513.

(15) Cook, N. D., Upperton, K. P., Challis, B. C., and Peters, T. J. (1987) The donor specificity and kinetics of the hydrolysis reaction of $\gamma$-glutamyltransferase. Biochim. Biophys. Acta 914, 240-245.

(16) Castonguay, R., Halim, D., Morin, M., Furtos, A., Lherbet, C., Bonneil, E., Thibault, P., and Keillor, J. W. (2007) Kinetic characterization and identification of the acylation and glycosylation sites of recombinant human $\gamma$-glutamyltranspeptidase. Biochemistry 46, $12253-12262$.

(17) Menard, A., Castonguay, R., Lherbet, C., Rivard, C., Roupioz, Y., and Keillor, J. W. (2001) Nonlinear free energy relationship in the general-acid-catalyzed acylation of rat kidney $\gamma$-glutamyl transpeptidase by a series of $\gamma$-glutamyl anilide substrate analogues. Biochemistry 40 , $12678-12685$.

(18) Han, L., Hiratake, J., Tachi, N., Suzuki, H., Kumagai, H., and Sakata, K. (2006) $\gamma$-(Monophenyl)phosphono glutamate analogues as mechanism-based inhibitors of $\gamma$-glutamyl transpeptidase. Bioorg. Med. Chem. 14, 6043-6054.

(19) Suzuki, H., Kumagai, H., and Tochikura, T. (1986) $\gamma$-Glutamyltranspeptidase from Escherichia coli K-12: Purification and properties. J. Bacteriol. 168, 1325-1331.

(20) Okada, T., Suzuki, H., Wada, K., Kumagai, H., and Fukuyama, K. (2006) Crystal structures of $\gamma$-glutamyltranspeptidase from Escherichia coli, a key enzyme in glutathione metabolism, and its reaction intermediate. Proc. Natl. Acad. Sci. U.S.A. 103, 6471-6476.

(21) Boanca, G., Sand, A., Okada, T., Suzuki, H., Kumagai, H., Fukuyama, K., and Barycki, J. J. (2007) Autoprocessing of Helicobacter pylori $\gamma$-glutamyltranspeptidase leads to the formation of a threoninethreonine catalytic dyad. J. Biol. Chem. 282, 534-541.

(22) Morrow, A. L., Williams, K., Sand, A., Boanca, G., and Barycki, J. J. (2007) Characterization of Helicobacter pylori $\gamma$-glutamyltranspeptidase reveals the molecular basis for substrate specificity and a critical role for the tyrosine 433-containing loop in catalysis. Biochemistry 46, 13407-13414.

(23) Williams, K., Cullati, S., Sand, A., Biterova, E. I., and Barycki, J. J. (2009) Crystal structure of acivicin-inhibited $\gamma$-glutamyltranspeptidase reveals critical roles for its $\mathrm{C}$-terminus in autoprocessing and catalysis. Biochemistry 48, 2459-2467.

(24) Wada, K., Hiratake, J., Irie, M., Okada, T., Yamada, C., Kumagai, H., Suzuki, H., and Fukuyama, K. (2008) Crystal structures of Escherichia coli $\gamma$-glutamyltranspeptidase in complex with azaserine and acivicin: Novel mechanistic implication for inhibition by glutamine antagonists. J. Mol. Biol. 380, 361-372.

(25) Wu, R., Richter, S., Zhang, R. G., Anderson, V. J., Missiakas, D., and Joachimiak, A. (2009) Crystal structure of Bacillus anthracis transpeptidase enzyme CapD. J. Biol. Chem. 284, 24406-24414.

(26) Thompson, G. A., and Meister, A. (1977) Interrelationships between the binding sites for amino acids, dipeptides, and $\gamma$-glutamyl donors in $\gamma$-glutamyl transpeptidase. J. Biol. Chem. 252, 6792-6798.

(27) Suzuki, H., Izuka, S., Minami, H., Miyakawa, N., Ishihara, S., and Kumagai, H. (2003) Use of bacterial $\gamma$-glutamyltranspeptidase for enzymatic synthesis of $\gamma$-D-glutamyl compounds. Appl. Environ. Microbiol. 69, 6399-6404.

(28) Ikeda, Y., Fujii, J., Taniguchi, N., and Meister, A. (1995) Expression of an active glycosylated human $\gamma$-glutamyl transpeptidase mutant that lacks a membrane anchor domain. Proc. Natl. Acad. Sci. U.S.A. 92, 126-130.

(29) Ikeda, Y., Fujii, J., Anderson, M. E., Taniguchi, N., and Meister, A. (1995) Involvement of Ser-451 and Ser-452 in the catalysis of human $\gamma$-glutamyl transpeptidase. J. Biol. Chem. 270, 22223-22228.

(30) Boanca, G., Sand, A., and Barycki, J. J. (2006) Uncoupling the enzymatic and autoprocessing activities of Helicobacter pylori $\gamma$-glutamyltranspeptidase. J. Biol. Chem. 281, 19029-19037.

(31) Chittur, S. V., Klem, T. J., Shafer, C. M., and Davisson, V. J. (2001) Mechanism for acivicin inactivation of triad glutamine amidotransferases. Biochemistry 40, 876-887.

(32) London, R. E., and Gabel, S. A. (2001) Development and evaluation of a boronate inhibitor of $\gamma$-glutamyl transpeptidase. Arch. Biochem. Biophys. 385, 250-258.

(33) Han, L., Hiratake, J., Kamiyama, A., and Sakata, K. (2007) Design, synthesis, and evaluation of $\gamma$-phosphono diester analogues of glutamate as highly potent inhibitors and active site probes of $\gamma$-glutamyl transpeptidase. Biochemistry 46, 1432-1447.

(34) King, J. B., West, M. B., Cook, P. F., and Hanigan, M. H. (2009) A novel, species-specific class of uncompetitive inhibitors of $\gamma$-glutamyl transpeptidase. J. Biol. Chem. 284, 9059-9065. 
(35) Nielsen, H., Engelbrecht, J., Brunak, S., and von, H. G. (1997) Identification of prokaryotic and eukaryotic signal peptides and prediction of their cleavage sites. Protein Eng. 10, 1-6.

(36) Chabot, D. J., Scorpio, A., Tobery, S. A., Little, S. F., Norris, S. L., and Friedlander, A. M. (2004) Anthrax capsule vaccine protects against experimental infection. Vaccine 23, 43-47.

(37) Keillor, J. W., Castonguay, R., and Lherbet, C. (2005) $\gamma$-Glutamyl transpeptidase substrate specificity and catalytic mechanism. Methods Enzymol. 401, 449-467.

(38) Anderson, M. E., Allison, R. D., and Meister, A. (1982) Interconversion of leukotrienes catalyzed by purified $\gamma$-glutamyl transpeptidase: Concomitant formation of leukotriene D4 and $\gamma$-glutamyl amino acids. Proc. Natl. Acad. Sci. U.S.A. 79, 10881091.

(39) Sali, A., Potterton, L., Yuan, F., van Vlijmen, H., and Karplus, M. (1995) Evaluation of comparative protein modeling by MODELLER. Proteins 23, 318-326.

(40) Sippl, M. J. (1993) Recognition of errors in three-dimensional structures of proteins. Proteins 17, 355-362.

(41) Laskowski, R. A., Macarthur, M. W., Moss, D. S., and Thornton, J. M. (1993) Procheck: A Program to Check the Stereochemical Quality of Protein Structures. J. Appl. Crystallogr. 26, 283-291.

(42) Case, D. A., Cheatham, T. E. III, Darden, T., Gohlke, H., Luo, R., Merz, K. M. Jr., Onufriev, A., Simmerling, C., Wang, B., and Woods, R. J. (2005) The Amber biomolecular simulation programs. J. Comput. Chem. 26, 1668-1688.

(43) Jiang, X., Kumar, K., Hu, X., Wallqvist, A., and Reifman, J. (2008) DOVIS 2.0: An efficient and easy to use parallel virtual screening tool based on AutoDock 4.0. Chem. Cent. J. 2, 18.

(44) Onufriev, A., Bashford, D., and Case, D. A. (2000) Modification of the generalized Born model suitable for macromolecules. J. Phys. Chem. B 104, 3712-3720.

(45) Rosenfield, R. E., Parthasarathy, R., and Dunitz, J. D. (1977) Directional Preferences of Nonbonded Atomic Contacts with Divalent Sulfur. 1. Electrophiles and Nucleophiles. J. Am. Chem. Soc. 99, 48604862.

(46) Gregoret, L. M., Rader, S. D., Fletterick, R. J., and Cohen, F. E. (1991) Hydrogen bonds involving sulfur atoms in proteins. Proteins 9, 99-107.

(47) Khavrutskii, I. V., Price, D. J., Lee, J., and Brooks, C. L. III (2007) Conformational change of the methionine 20 loop of Escherichia coli dihydrofolate reductase modulates $\mathrm{pKa}$ of the bound dihydrofolate. Protein Sci. 16, 1087-1100.

(48) Wada, K., Irie, M., Suzuki, H., and Fukuyama, K. (2010) Crystal structure of the halotolerant $\gamma$-glutamyltranspeptidase from Bacillus subtilis in complex with glutamate reveals a unique architecture of the solvent-exposed catalytic pocket. FEBS J. 277, 1000-1009.

(49) Xu, K., and Strauch, M. A. (1996) Identification, sequence, and expression of the gene encoding $\gamma$-glutamyltranspeptidase in Bacillus subtilis. J. Bacteriol. 178, 4319-4322.

(50) Ikeda, Y., Fujii, J., Taniguchi, N., and Meister, A. (1995) Human $\gamma$-glutamyl transpeptidase mutants involving conserved aspartate residues and the unique cysteine residue of the light subunit. J. Biol. Chem. 270, 12471-12475.

(51) Allison, R. D., and Meister, A. (1981) Evidence that transpeptidation is a significant function of $\gamma$-glutamyl transpeptidase. J. Biol. Chem. 256, 2988-2992.

(52) Petrey, D., Xiang, Z., Tang, C. L., Xie, L., Gimpelev, M., Mitros, T., Soto, C. S., Goldsmith-Fischman, S., Kernytsky, A., Schlessinger, A., Koh, I. Y., Alexov, E., and Honig, B. (2003) Using multiple structure alignments, fast model building, and energetic analysis in fold recognition and homology modeling. Proteins 53 (Suppl. 6), 430-435. 